\title{
Mechanism of miR-132-3p Promoting Neuroinflammation and Dopaminergic Neurodegeneration in Parkinson's Disease
}

\author{
Xin Gong, Mengyi Huang, and $\mathbb{C}^{-}$Lei Chen
}

https://doi.org/10.1523/ENEURO.0393-21.2021

Department of Neurosurgery, Hunan Provincial People's Hospital, The First Affiliated Hospital of Hunan Normal University, Changsha, Hunan 410005, People's Republic of China

\section{Visual Abstract}

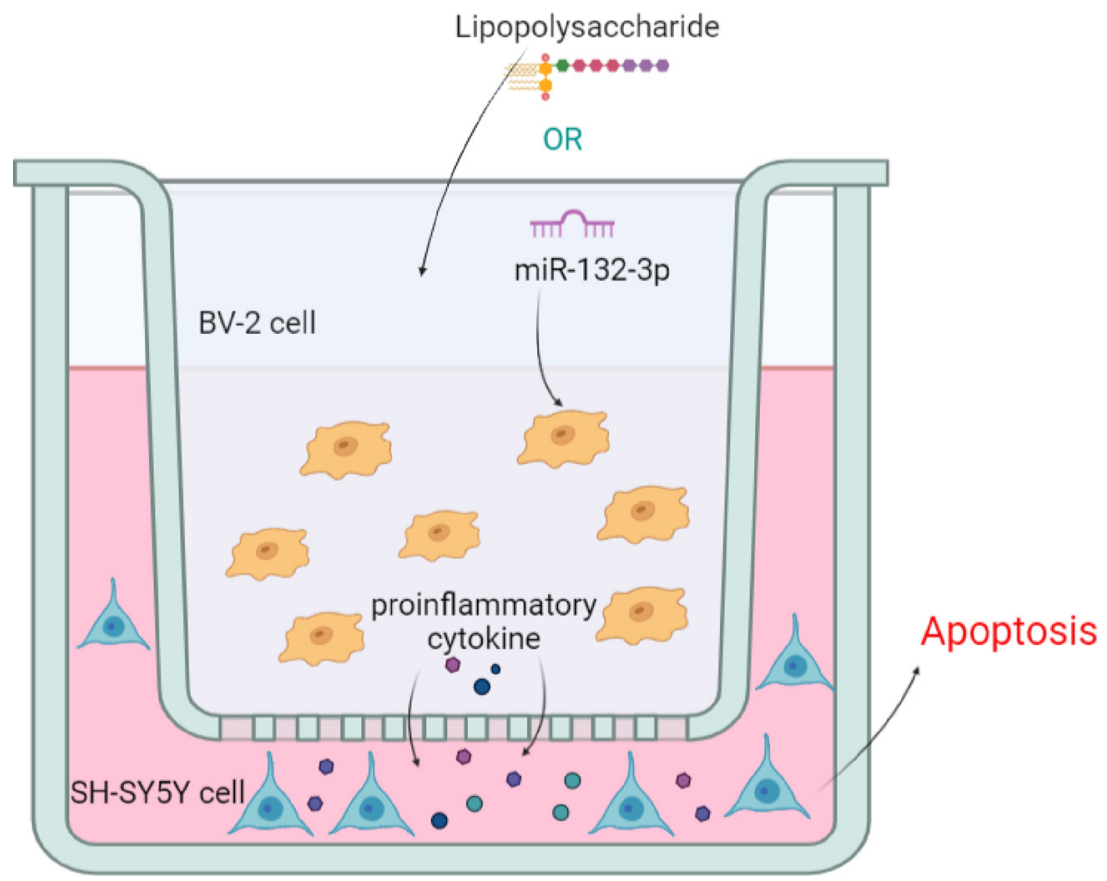

The major pathology in Parkinson's disease (PD) is neuron injury induced by degeneration of dopaminergic neurons and the activation of microglial cells. The objective of this study is to determine the effect and mechanism of miR-132-3p in regulating neuroinflammation and the degeneration of dopaminergic neuron in PD. The

\section{Significance Statement}

The purpose of this study is to explore the possible effect and mechanism of miR-132-3p/GLRX on neuroinflammation and the degeneration of dopaminergic neuron in Parkinson's disease (PD). This is important as no effective treatment is available to cure PD and better understanding on how neuroinflammation and degeneration of dopaminergic neurons was regulated in PD will facilitate the proposal of therapeutic strategy. Future study should further validate the role and mechanism of miR-132-3p in regulating PD through GLRX before miR-132-3p can be proposed as a novel therapeutic target. 
expressions of miR-132-3p in brain tissues of PD patients, lipopolysaccharide (LPS)-induced BV-2 cells and 1methyl-4-phenyl-1,2,3,6-tetrahydropyridine (MPTP)-induced PD mouse models were detected. The effect of miR-132-3p and GLRX in cell viability, apoptosis and inflammation was verified in BV-2 cells. The activation of Iba1 in substantia nigra pars compacta (SNc) and the loss of tyrosine hydroxylase were detected in PD mouse models and the mobility of mouse models was assessed as well. The targeting relationship between miR-132$3 p$ and GLRX was confirmed by RNA immunoprecipitation (RIP) and dual luciferase reporter gene assay. Elevated expression of miR-132-3p and decreased expression of GLRX were found in PD patients and cells models. Overexpression of miR-132-3p can induce activation of microglial cells, which can be reversed by GLRX overexpression. Collected evidence in both cell model and mouse models showed the effect of miR$132-3 p$ in enhancing the activation of microglial cells and the loss of microglia cells, which was achieved by mediating GLRX.

Key words: Parkinson's disease; MiR-132-3p; neuroinflammation; dopaminergic neuron; GLRX; MPTP

\section{Introduction}

Parkinson's disease (PD) is a neurodegenerative disease in elderly caused by degeneration of dopaminergic neurons mainly in the substantia nigra pars compacta (SNc), a region of the midbrain (Sveinbjornsdottir, 2016; Alizadeh et al., 2019). Severe locomotor deficit, such as freezing of gait, is a typical phenomenon of PD, which refers to intermittent walking disturbance during walk initiation and turning (Strubberg and Madison, 2017). The etiology of $\mathrm{PD}$ is probably multifactorial and currently there is no available treatment that can attenuate the neurodegenerative process of the disease. Therefore, a clearer understanding of mechanizing driving PD progression would be beneficial for the proposal of therapeutic approach. A variety of molecular mechanisms that likely contribute to neuronal cell death have been described previously, including $\alpha$-synuclein aggregation, mitochondrial dysfunction and noxious oxidant stress (Milanese et al., 2021). In addition, neuroinflammation is one of the hallmarks of PD and may induce the degeneration of midbrain dopamine neurons (Krashia et al., 2019). Therefore, therapeutic intervention would be a potential strategy to alleviate the progression of PD by interfering with neuroinflammation and degeneration of dopaminergic neurons.

MicroRNA (miRNA) is an endogenic RNA comprised of 21-24 nt, which controls gene transcription in combination with the $3^{\prime}$-untranslated region (UTR) of multiple targeted mRNAs (Li et al., 2017; Angelopoulou et al., 2019).

Received September 24, 2021; accepted December 2, 2021; First published January 4, 2022.

The authors declare no competing financial interests.

Author contributions: X.G. and L.C. designed research; X.G., M.H., and L.C. performed research; X.G., M.H., and L.C. contributed unpublished reagents/ analytic tools; X.G., M.H., and L.C. analyzed data; X.G., M.H., and L.C. wrote the paper.

This work was funded by the Hunan Provincial Natural Science Foundation Grant No. 2019JJ50322 and the Science Research Planning Program of Health and Family Planning Commission of Hunan Province Grant No. C2019054.

Acknowledgements: We thank all the contributors and participants. Correspondence should be addressed to Lei Chen at798642313@qq.com. https://doi.org/10.1523/ENEURO.0393-21.2021 Copyright (C) 2022 Gong et al.

This is an open-access article distributed under the terms of the Creative Commons Attribution 4.0 International license, which permits unrestricted use, distribution and reproduction in any medium provided that the original work is properly attributed.
About 1900 miRNAs that can be encoded by human genome (Strubberg and Madison, 2017). Among these miRNAs, miR-132 has been frequently mentioned in many researches for its increased expression in neurons and for its implication in various neurodegenerative disorders. For example, the enhanced expression of miR-132-3p is related to chronic neuropathic pain (Leinders et al., 2016). MiR-132/Nurr1 axis was reported to have certain relationship with PD progression (Yang et al., 2019). Moreover, neuronal inflammation-induced epilepsy may be attenuated by miR-132 by targeting TRAF6, along with inactivation of NF- $\kappa \mathrm{B}$ and MEK/ERK pathways (Ji et al., 2018). MiR-132 is positively associated with dopaminergic neuronal death (Qazi et al., 2021). Evidence in previous study pointed out that microglial cells medicated neuroinflammation triggered the cascade of inflammatory events leading to neuronal degeneration (Hirsch and Hunot, 2009; Bassani et al., 2015; Ye et al., 2018). However, it remains unclear how miR-132-3p is involved in neuroinflammation and dopaminergic neurodegeneration in PD.

GLRX is a small protein that catalyzes the glutathionedependent disulfide oxidoreduction reactions in a coupled system (Wang et al., 2019). In models of PD, deficiency of GLRX aggravates neurodegeneration (Johnson et al., 2015). Meanwhile, previous study addressed that suppression of GLRX contributes to PD-relevant motor deficits and dopaminergic degeneration in mice (Verma et al., 2020). Therefore, we speculated GLRX also has certain role to play in neuroinflammation and dopaminergic neurodegeneration in PD. Online software predicted that miR-132-3p was identified as an upstream regulatory factor of GLRX. In this regard, this study aims to investigate the mechanism by which miR-132-3p regulates neuroinflammation and dopaminergic neuron degeneration in PD. Hence, exploring the interactions between miR-132-3p/GLRX, dopaminergic neurodegeneration and neuroinflammation may be of great importance for the proposal of a latent therapeutic alternative for PD.

The main aims of the present study were to determine (1) whether miR-132-3p expression level is significantly altered in patients with PD as compared with healthy controls; (2) whether miR-132-3p is responsible for microglial activation and neuronal injury; (3) whether miR-132-3p affects the dopaminergic neuron degeneration and neuroinflammation in PD mouse 
models; and (4) whether miR-132-3p intensifies PD by inhibiting GLRX.

\section{Materials and Methods}

\section{Collection of clinical brain tissues}

The study was conducted according to the Declaration of Helsinki. The study protocol concerning human was approved by the Ethics Committee of Hunan Provincial People's Hospital (No. 202004), and written informed consent from family members of included subjects was obtained. This study was not preregistered and no sample calculation was performed. After death, the midbrain tissues were obtained from five patients with PD (three males and two females, $55.8 \pm 7.09$ years old) and five healthy controls (three males and two females, $59.8 \pm 8.07$ years old) matched for age. The brain tissues were collected and stored in liquid nitrogen, in which the total RNA and total protein were stored at $-80^{\circ} \mathrm{C}$ refrigerator. The diagnosis of PD was performed by at least two or more experienced neurologists based on the clinical diagnostic criteria proposed by International Parkinson and Movement Disorder Society (Postuma et al., 2018). The included PD patients were excluded from secondary PD, tumor, or metabolic disturbance. The healthy controls were excluded from disorders related to nervous system.

\section{Cell culture}

The BV-2 microglial cells, human neuroblastoma cell line SH-SY5Y and human embryonic kidney (HEK)293T cells were supplied by American type Culture Collection (ATCC). The BV-2 and HEK293T cells were soaked in DMEM (Invitrogen), and SH-SY5Y cells were immersed in DMEM/Nutrient Mixture F-12 (DMEM/F12, Invitrogen). The culture medium contained $10 \%$ FBS, $100 \mathrm{U} / \mathrm{ml}$ penicillin, and $100 \mathrm{mg} / \mathrm{ml}$ streptomycin. Cell culture was kept at $37^{\circ} \mathrm{C}$ in a humidified atmosphere containing $5 \% \mathrm{CO}_{2}$. The inflammation of BV-2 cells was induced by $0.1 \mu \mathrm{g} / \mathrm{ml}$ lipopolysaccharide (LPS) for $24 \mathrm{~h}$. The cell passage of cell lines shall not exceed 10 times.

\section{Cell transfection}

The miR-132-3p mimic (miRNA mimic refers to a sequence that can simulate specific endogenous miRNA), mimic negative control (NC), miR-132-3p inhibitor (miRNA inhibitor refers to a sequence that can interfere with miRNA), inhibitor NC, overexpressing GLRX (GLRX) and pcDNA3.1 were synthesized and purchased from GenePharma. Cell transfection was conducted by using the Lipofectamine 3000 reagent (Invitrogen). The transfection dose of overexpression plasmids was $2 \mu \mathrm{g}$, and the dose of mimic and inhibitor was $50 \mathrm{~nm}$. Cells were treated by LPS for $24 \mathrm{~h}$ before following experiments were conducted.

\section{qRT-PCR}

TRIzol (Invitrogen) was employed to extract the total RNA of tissues or cells, and the reverse transcription was conducted by using the reverse transcription kit (TaKaRa). The expression of gene was quantitated by LightCycler 480 fluorescence quantitative PCR instrument (Roche),
Table 1: Primer sequence information

\begin{tabular}{ll}
\hline Name of primer & Sequences \\
\hline U6-F & CTCGCTTCGGCAGCACA \\
U6-R & AACGCTTCACGAATTTGGT \\
miR-132-3p -F & GCAACGTAACAGTCTACAGCC \\
miR-132-3p -R & CCAGTGCAGGGTCCGAGGTA \\
$\beta-$ Actin-F & TGTACCCAGGCATTGCTGAC \\
$\beta-$ Actin-R & AACGCAGCTCAGTAACAGTCC \\
GLRX-F & AGTTATAAAAGGGGTGGCAGAGT \\
GLRX-R & CCCCATGGTTAGGGGCAAAT \\
TNF- $\alpha-\mathrm{F}$ & AGGCACTCCCCCAAAAGATG \\
TNF- $\alpha-\mathrm{R}$ & CCACTTGGTGGTTTGGAGTG \\
IL-1 $\beta-\mathrm{F}$ & TGCCACCTTTGACAGTGATG \\
IL-1 $\beta-\mathrm{R}$ & AAGGTCCACGGGAAAGACAC \\
IL-6-F & CAACGATGATGCACTTGCAGA \\
IL-6-R & TGTGACTCCAGCTTATCTCTTGG \\
\hline
\end{tabular}

F: forward primer; R: reverse primer.

and reaction condition was instructed by the fluorescence quantitative PCR kit (SYBR Green Mix, Roche Diagnostics). The thermal cycle parameters were $95^{\circ} \mathrm{C}$ for $10 \mathrm{~s}$, followed by 45 cycles of $95^{\circ} \mathrm{C}$ for $5 \mathrm{~s}, 60^{\circ} \mathrm{C}$ for $10 \mathrm{~s}$, and $72^{\circ} \mathrm{C}$ for $10 \mathrm{~s}$. A final extension was conducted at $72^{\circ} \mathrm{C}$ for $5 \mathrm{~min}$. The quantification of mRNA was normalized to $\beta$-actin and miRNA to U6. The fold changes were calculated by the $2^{-\Delta \Delta \mathrm{Ct}}$ method. The formula is as follows: $\Delta \Delta \mathrm{Ct}=\left[\mathrm{Ct}_{\text {(target gene) }}-\mathrm{Ct}_{\text {(reference gene) }}\right]_{\text {experimental group }}-$ $\left[\mathrm{Ct}_{\text {(target gene) }}-\mathrm{Ct}_{\text {(reference gene) }}\right]_{\text {control group. All primers are }}$ shown in Table 1.

\section{Western blotting}

For collection of protein samples, RIPA lysis buffer (Beyotime Biotech) was used to treat cells or tissues. Following determination of protein concentration with a $\mathrm{BCA}$ kit, the proteins in the corresponding volume were mixed with loading buffer (Beyotime) and subjected to denaturation in a boiling-water bath for $3 \mathrm{~min}$. Electrophoresis was embarked at $80 \mathrm{~V}$ for $30 \mathrm{~min}$, and then for $1-2 \mathrm{~h}$ at 120 $\mathrm{V}$ when bromphenol blue reached the separation gel. The

Table 2: Abbreviation list

\begin{tabular}{ll}
\hline Abbreviations & Full names \\
\hline PD & Parkinson's disease \\
GLRX & Glutaredoxin \\
LPS & Lipopolysaccharide \\
qRT-RCR & Quantitative real-time polymerase chain reaction \\
ELISA & Enzyme-linked immunosorbent assay \\
RIP & RNA immunoprecipitation \\
MPTP & 1-Methyl-4-phenyl-1, 2, 3, 6-tetrahydropyridine \\
SNc & Substantia nigra compacta \\
TH & Tyrosine hydroxylase \\
DMEM & Dulbecco's modified eagle medium \\
PBS & Pphosphate buffer \\
Ago2 & Argonaute 2 \\
FBS & Fetal bovine serum \\
FITC & Fluorescein isothiocyanate \\
PI & Propidium iodide \\
FISH & Fluorescence in situ hybridization \\
RRID & Research Resource ldentifier \\
NC & negative control \\
\hline
\end{tabular}


Table 3: Reagents and materials

\begin{tabular}{|c|c|}
\hline Names & $\begin{array}{l}\text { RRIDs or catalog } \\
\text { number }\end{array}$ \\
\hline BV-2 cell & YB-ATCC-4255, ATCC \\
\hline SH-SY5Y cell & RRID:CVCL_0019 \\
\hline HEK293T cell & RRID:CVCL_0063 \\
\hline DMEM & 11054001, Gibco \\
\hline DMEM/F12 & 11330107, Gibco \\
\hline FBS & 16140, Invitrogen \\
\hline Penicillin/streptomycin & 15140148, Invitrogen \\
\hline LPS & L-4391, Sigma-Aldrich \\
\hline Lipofectamine 3000 & L3000150, Invitrogen \\
\hline TRlzol & 15596026, Invitrogen \\
\hline Reverse transcription kit & 6210A, TaKaRa \\
\hline SYBR Green Mix & 2015099, Roche \\
\hline RIPA Iysis buffer & P0013C, Beyotime \\
\hline BCA kit & P0012, Beyotime \\
\hline Protein loading buffer & P0015A, Beyotime \\
\hline$\beta$-Actin antibody & RRID:AB_306371 \\
\hline GLRX antibody & RRID:AB_880242 \\
\hline Film development kit & P0019, Beyotime \\
\hline TNF- $\alpha$ & DY410, R\&D \\
\hline $\mathrm{IL}-1 \beta$ & SMLBOOC, R\&D \\
\hline IL-6 & SM6000B, R\&D \\
\hline CCK-8 kit & CK04, Dojindo \\
\hline Annexin V-FITC cell apoptosis kit & C1062L, Beyotime \\
\hline PBS & C0221A, Beyotime \\
\hline$A+G$ beads & P2108, Beyotime \\
\hline Ago2 antibody & RRID:AB_867543 \\
\hline $\lg G$ antibody & RRID:AB_2687931 \\
\hline pGL3-Promoter & E1761, Promega \\
\hline pRL-TK & E2241, Promega \\
\hline $\begin{array}{l}\text { Dual-Luciferase Reporter Assay } \\
\text { System }\end{array}$ & E1910, Promega \\
\hline MPTP & M0896, Sigma-Aldrich \\
\hline $4 \%$ paraformaldehyde & P0099, Beyotime \\
\hline Proteinase $\mathrm{K}$ & ST535, Beyotime \\
\hline Tyrosine hydroxylase antibody & RRID:AB_2801410 \\
\hline Iba1 antibody & RRID:AB_2636859 \\
\hline DAPI & C1002, Beyotime \\
\hline MiRNA mimic & B02003, GenePharma \\
\hline MiRNA inhibitor & B03001, GenePharma \\
\hline Gene overexpression plasmid & C05001, GenePharma \\
\hline MiRNA antagomir & B05001, GenePharma \\
\hline
\end{tabular}

proteins were transferred onto membranes at $300 \mathrm{~mA}$ for $60 \mathrm{~min}$ in an ice bath. Then, the membranes were rinsed 12 min with washing solution and inactivated in the blocking solution at room temperature for $60 \mathrm{~min}$, or sealed overnight at $4^{\circ} \mathrm{C}$. Following incubation with the primary antibodies against $\beta$-actin (ab8226, $1 \mu \mathrm{g} / \mathrm{ml}$ ) and GLRX (ab45953, 1:250; Abcam) at room temperature in a shaking table for $1 \mathrm{~h}$, the membranes were washed with the washing solution for $3 \times 10 \mathrm{~min}$ and incubated with the secondary antibody for $1 \mathrm{~h}$ at room temperature. The membranes were washed thrice for $10 \mathrm{~min}$ and exposed to developing liquid for color development. Then, the membranes were observed in chemiluminecence imaging analysis system (Gel Doc XR, Bio-Rad).

\section{ELISA}

The ELISA kit (R\&D Systems) was adopted to determine the contents of TNF- $\alpha, \mathrm{IL}-6$, and $\mathrm{IL}-1 \beta$ in the cell supernatant. All operations were performed in accordance with the instructions of the ELISA kit.

\section{Coculture of BV-2 and SH-SY5Y cells}

The effect of microglial activation on SH-SY5Y cells was studied by coculture of BV-2 cell supernatant and $\mathrm{SH}-\mathrm{SY} 5 \mathrm{Y}$ cells. The supernatant of BV-2 cells in each group was collected and filtered with a $0.45-\mu \mathrm{m}$ filter. $\mathrm{SH}-$ SY5Y cells were seeded onto the six-well plates for cell culture till the density of SH-SY $5 Y$ cells reached $70 \%$. The supernatant of BV-2 cells and DMEM/F12 containing $10 \%$ FBS were mixed at a ratio of $1: 1$, and the mixture was co-cultured with $\mathrm{SH}-\mathrm{SY} 5 \mathrm{Y}$ cells for $24 \mathrm{~h}$.

\section{CCK-8 assay}

The SH-SY5Y cells were seeded onto 96-well plates, and cells in each well received $100-\mu$ l prediluted cell suspension $\left(1 \times 10^{5}\right.$ cells $\left./ \mathrm{ml}\right)$. Twenty-four hours later, $\mathrm{SH}$ SY5Y cells were grown in conditioned medium of BV-2 cells for $24 \mathrm{~h}$. The experiment was designed with three replicates. Ten microliters of CCK-8 solution (Dojindo) was added to each well for $2 \mathrm{~h}$ of incubation. The optical density (OD) at 450-nm wavelength was assessed.

\section{Flow cytometry}

After SH-SY5Y cells $\left(10^{5}\right.$ cells $\left./ \mathrm{ml}\right)$ were incubated with conditioned medium of BV-2 cells for $24 \mathrm{~h}, 3-\mathrm{ml}$ cell suspension from each sample was transferred into a $10-\mathrm{ml}$ centrifuge tube for $5 \mathrm{~min}$ of centrifugation at $500 \mathrm{rpm}$. After removal of culture medium, cells were washed with PBS and centrifuged at $500 \mathrm{rpm}$ for $5 \mathrm{~min}$. The supernatant was discarded. Then, cells were resuspended in $100 \mu \mathrm{l}$ of binding buffer, and then gently mixed with $5-\mu \mathrm{l}$ Annexin V-fluorescein isothiocyanate (FITC) and $5 \mu \mathrm{l} \mathrm{PI} \mathrm{for}$ incubation for $15 \mathrm{~min}$ in the dark. The fluorescence of FITC and PI was examined by flow cytometry, and the apoptosis rate was analyzed.

\section{RNA immunoprecipitation (RIP)}

Following wash twice with precooled PBS, cells were centrifuged at $1500 \mathrm{rpm}$ for $5 \mathrm{~min}$ and lysed with equivoluminal RIP lysis buffer. The magnetic beads were resuspended in $100-\mu$ I RIP Wash buffer followed by 30 min of incubation with 5- $\mu \mathrm{g}$ antibody against Ago2 (ab186733, 1:30, Abcam) or IgG (ab172730, negative control) at room temperature. Cells in the centrifuge tube were placed on a magnetic separation rack to discard the supernatant. Following incubation with 500- $\mu$ I RIP Wash buffer for vortex oscillation twice, cells were given $500 \mu$ l of RIP Wash buffer for vortex oscillation and placed on ice. The magnetic bead tube was transferred to the magnetic separation rack, and the supernatant was removed. After that, cells in each tube received $900 \mu$ l of RIP immunoprecipitation buffer. Cell lysates were centrifuged at $14,000 \mathrm{rpm}$ at $4^{\circ} \mathrm{C}$ for $10 \mathrm{~min}$, and $100 \mu \mathrm{l}$ of supernatant was pipetted into the magnetic bead-antibody complex for incubation overnight at $4^{\circ} \mathrm{C}$. The complex processed centrifugation with supernatant removed. Then, the centrifuge tube received $500 \mu$ l of RIP Wash buffer for vortex oscillation and 
Table 4: Statistical summary and analysis methods

\begin{tabular}{|c|c|c|c|c|c|c|c|c|}
\hline Figure reported & $N$ & $\begin{array}{l}\text { Normal } \\
\text { distribution }\end{array}$ & Statistic & $\begin{array}{l}\text { Statistic value } \\
\text { (df) }\end{array}$ & $p$ value & $\begin{array}{l}\text { Variance } \\
\text { source }\end{array}$ & $\begin{array}{l}\text { Post hoc } \\
\text { test }\end{array}$ & Post hoc $p$ \\
\hline $1 A, \mathrm{PD}$ vs control & 5 & Yes & Unpaired $t$ test & $t_{(8)}=2.644$ & 0.0295 & Difference & & \\
\hline $1 B, \mathrm{PD}$ vs control & 5 & Yes & Unpaired $t$ test & $t_{(8)}=3.418$ & 0.0091 & Difference & & \\
\hline $1 C, \mathrm{PD}$ vs control & 5 & Yes & Unpaired $t$ test & $t_{(8)}=2.449$ & 0.0400 & Difference & & \\
\hline $2 A$ & 3 & Yes & One-way ANOVA & $F_{(3,8)}=41.01$ & $<0.0001$ & Treatment & & \\
\hline $2 A$, PBS vs LPS & 3 & Yes & & & & & Tukey & 0.0025 \\
\hline $2 A$, LPS + inhibitor NC vs LPS + miR-132-3p inhibitor & 3 & Yes & & & & & Tukey & $<0.0001$ \\
\hline $2 B$, TNF- $\alpha$ & 3 & Yes & One-way ANOVA & $F_{(3,8)}=62.48$ & $<0.0001$ & Treatment & & \\
\hline $2 B$, TNF- $\alpha$, PBS vs LPS & 3 & Yes & & & & & Tukey & $<0.0001$ \\
\hline $2 B$, TNF- $\alpha$, LPS + inhibitor NC vs LPS + miR-132-3p inhibitor & 3 & Yes & & & & & Tukey & 0.001 \\
\hline $2 B, \mathrm{IL}-1 \beta$ & 3 & Yes & One-way ANOVA & $F_{(3,8)}=68.03$ & $<0.0001$ & Treatment & & \\
\hline $2 B, \mathrm{IL}-1 \beta, \mathrm{PBS}$ vs LPS & 3 & Yes & & & & & Tukey & $<0.0001$ \\
\hline $2 B, I L-1 \beta$, LPS + inhibitor NC vs LPS + miR-132-3p inhibitor & 3 & Yes & & & & & Tukey & 0.001 \\
\hline $2 B, I L-6$ & 3 & Yes & One-way ANOVA & $F_{(3,8)}=55.19$ & $<0.0001$ & Treatment & & \\
\hline $2 B, I L-6$, PBS vs LPS & 3 & Yes & & & & & Tukey & $<0.0001$ \\
\hline $2 B$, IL-6, LPS + inhibitor NC vs LPS + miR-132-3p inhibitor & 3 & Yes & & & & & Tukey & 0.005 \\
\hline $2 C$, TNF- $\alpha$ & 3 & Yes & One-way ANOVA & $F_{(3,8)}=38.98$ & $<0.0001$ & Treatment & & \\
\hline $2 C$, TNF- $\alpha$, PBS vs LPS & 3 & Yes & & & & & Tukey & $<0.0001$ \\
\hline $2 C$, TNF- $\alpha$, LPS + inhibitor NC vs LPS + miR-132-3p inhibitor & 3 & Yes & & & & & Tukey & 0.0059 \\
\hline $2 C, \mathrm{IL}-1 \beta$ & 3 & Yes & One-way ANOVA & $F_{(3,8)}=70.75$ & $<0.0001$ & Treatment & & \\
\hline $2 C, \mathrm{IL}-1 \beta, \mathrm{PBS}$ vs LPS & 3 & Yes & & & & & Tukey & $<0.0001$ \\
\hline $2 C$, IL- $1 \beta$, LPS + inhibitor NC vs LPS + miR-132-3p inhibitor & 3 & Yes & & & & & Tukey & 0.001 \\
\hline $2 C$, IL-6 & 3 & Yes & One-way ANOVA & $F_{(3,8)}=86.8$ & $<0.0001$ & Treatment & & \\
\hline $2 C$, IL-6, PBS vs LPS & 3 & Yes & & & & & Tukey & $<0.0001$ \\
\hline $2 C$, IL-6, LPS + inhibitor NC vs LPS + miR-132-3p inhibitor & 3 & Yes & & & & & Tukey & 0.001 \\
\hline $3 A$ & 3 & Yes & One-way ANOVA & $F_{(2,6)}=343.2$ & $<0.0001$ & Treatment & & \\
\hline $3 A$, mimic NC vs miR-132-3p mimic & 3 & Yes & & & & & Tukey & $<0.0001$ \\
\hline $3 B$, TNF- $\alpha$ & 3 & Yes & One-way ANOVA & $F_{(2,6)}=47.96$ & 0.0002 & Treatment & & \\
\hline $3 B$, TNF- $\alpha$, mimic NC vs miR-132-3p mimic & 3 & Yes & & & & & Tukey & 0.0003 \\
\hline $3 B,-\mathrm{IL}-1 \beta$ & 3 & Yes & One-way ANOVA & $F_{(2,6)}=51.95$ & 0.0002 & Treatment & & \\
\hline $3 B-\mathrm{IL}-1 \beta$, mimic NC vs miR-132-3p mimic & 3 & Yes & & & & & Tukey & 0.0003 \\
\hline $3 B, \mathrm{IL}-6$ & 3 & Yes & One-way ANOVA & $F_{(2,6)}=35$ & 0.0005 & Treatment & & \\
\hline $3 B, I L-6$, mimic NC vs miR-132-3p mimic & 3 & Yes & & & & & Tukey & 0.0016 \\
\hline $3 C$, TNF- $\alpha$ & 3 & Yes & One-way ANOVA & $F_{(2,6)}=69.99$ & $<0.0001$ & Treatment & & \\
\hline $3 C$, TNF- $\alpha$, mimic NC vs miR-132-3p mimic & 3 & Yes & & & & & Tukey & 0.0002 \\
\hline $3 C, \mathrm{IL}-1 \beta$ & 3 & Yes & One-way ANOVA & $F_{(2,6)}=188.5$ & $<0.0001$ & Treatment & & \\
\hline $3 C, \mathrm{IL}-1 \beta$, mimic NC vs miR-132-3p mimic & 3 & Yes & & & & & Tukey & $<0.0001$ \\
\hline $3 C$, IL-6 & 3 & Yes & One-way ANOVA & $F_{(2,6)}=152.1$ & $<0.0001$ & Treatment & & \\
\hline $3 C$, IL-6, mimic NC vs miR-132-3p mimic & 3 & Yes & & & & & Tukey & $<0.0001$ \\
\hline $4 A$ & 3 & Yes & One-way ANOVA & $F_{(3,8)}=68.62$ & $<0.0001$ & Treatment & & \\
\hline $4 A$, PBS vs LPS & 3 & Yes & & & & & Tukey & $<0.0001$ \\
\hline $4 A$, LPS + inhibitor NC vs LPS + miR-132-3p inhibitor & 3 & Yes & & & & & Tukey & 0.0050 \\
\hline $4 B$ & 3 & Yes & One-way ANOVA & $F_{(3,8)}=114.1$ & $<0.0001$ & Treatment & & \\
\hline $4 B$, PBS vs LPS & 3 & Yes & & & & & Tukey & $<0.0001$ \\
\hline $4 B$, LPS + inhibitor NC vs LPS + miR-132-3p inhibitor & 3 & Yes & & & & & Tukey & $<0.0001$ \\
\hline $4 C$ & 3 & Yes & One-way ANOVA & $F_{(2,6)}=9.25$ & 0.0147 & Treatment & & \\
\hline 4C, mimic NC vs miR-132-3p mimic & 3 & Yes & & & & & Tukey & 0.0461 \\
\hline $4 D$ & 3 & Yes & One-way ANOVA & $F_{(2,6)}=17.59$ & 0.0031 & Treatment & & \\
\hline $4 D$, mimic NC vs miR-132-3p mimic & 3 & Yes & & & & & Tukey & 0.0040 \\
\hline $5 A$ & 3 & Yes & One-way ANOVA & $F_{(3,8)}=35.92$ & $<0.0001$ & Treatment & & \\
\hline $5 A$, inhibitor NC vs miR-132-3p inhibitor & 3 & Yes & & & & & Tukey & 0.006 \\
\hline $5 A$, mimic NC vs miR-132-3p mimic & 3 & Yes & & & & & Tukey & 0.0089 \\
\hline $5 B$ & 3 & Yes & One-way ANOVA & $F_{(3,8)}=13.63$ & 0.0005 & Treatment & & \\
\hline $5 B$, inhibitor NC vs miR-132-3p inhibitor & 3 & Yes & & & & & Tukey & 0.01 \\
\hline $5 B$, mimic NC vs miR-132-3p mimic & 3 & Yes & & & & & Tukey & 0.0382 \\
\hline $5 C, \operatorname{miR}-132-3 p$ & 3 & Yes & One-way ANOVA & $F_{(3,8)}=115.1$ & $<0.0001$ & Treatment & & \\
\hline 5C, miR-132-3p, IgG vs Ago2 & 3 & Yes & & & & & Tukey & $<0.0001$ \\
\hline $5 C$, GLRX & 3 & Yes & One-way ANOVA & $F_{(3,8)}=126.1$ & $<0.0001$ & Treatment & & \\
\hline $5 C$, GLRX, IgG vs Ago2 & 3 & Yes & & & & & Tukey & $<0.0001$ \\
\hline $5 D$, PBS vs LPS & 3 & Yes & Two-way ANOVA & $F_{(2,12)}=12.1$ & 0.0013 & Interaction & & \\
\hline $5 D$, PBS vs LPS & 3 & Yes & Two-way ANOVA & $F_{(2,12)}=168.8$ & $<0.0001$ & Main effect & & \\
\hline $5 F$ & 3 & Yes & $\begin{array}{l}\text { One-way ANOVA } \\
\text { (Continued) }\end{array}$ & $F_{(3,8)}=9.623$ & 0.0050 & Treatment & & \\
\hline
\end{tabular}


Table 4: Continued

\begin{tabular}{|c|c|c|c|c|c|c|c|c|}
\hline Figure reported & $N$ & $\begin{array}{l}\text { Normal } \\
\text { distribution }\end{array}$ & Statistic & $\begin{array}{l}\text { Statistic value } \\
\text { (df) }\end{array}$ & $p$ value & $\begin{array}{l}\text { Variance } \\
\text { source }\end{array}$ & $\begin{array}{l}\text { Post hoc } \\
\text { test }\end{array}$ & Post hoc $p$ \\
\hline $5 F$, wt-GLRX + mimic NC vs wt-GLRX + miR-132-3p mimic & 3 & Yes & & & & & Tukey & 0.0044 \\
\hline $6 A$ & 3 & Yes & One-way ANOVA & $F_{(2,6)}=75.16$ & $<0.0001$ & Treatment & & \\
\hline $6 A$, mimic NC vs miR-132-3p mimic & 3 & Yes & & & & & Tukey & 0.002 \\
\hline $6 A$, miR-132-3p mimic vs miR-132-3p mimic + GLRX & 3 & Yes & & & & & Tukey & $<0.0001$ \\
\hline $6 B$ & 3 & Yes & One-way ANOVA & $F_{(2,6)}=36.68$ & 0.0004 & Treatment & & \\
\hline $6 B$, mimic NC vs miR-132-3p mimic & 3 & Yes & & & & & Tukey & 0.0165 \\
\hline $6 B$, miR-132-3p mimic vs miR-132-3p mimic + GLRX & 3 & Yes & & & & & Tukey & 0.004 \\
\hline $6 C$, TNF- $\alpha$ & 3 & Yes & One-way ANOVA & $F_{(2,6)}=43.7$ & 0.0003 & Treatment & & \\
\hline $6 C$, TNF- $\alpha$, mimic NC vs miR-132-3p mimic & 3 & Yes & & & & & Tukey & 0.0003 \\
\hline $6 C$, TNF- $\alpha$, miR-132-3p mimic vs miR-132-3p mimic + GLRX & 3 & Yes & & & & & Tukey & 0.001 \\
\hline $6 C, \mathrm{IL}-1 \beta$ & 3 & Yes & One-way ANOVA & $F_{(2,6)}=38.76$ & 0.0004 & Treatment & & \\
\hline $6 C, \mathrm{IL}-1 \beta$, mimic NC vs miR-132-3p mimic & 3 & Yes & & & & & Tukey & 0.0004 \\
\hline $6 C$, IL-1 $\beta$, miR-132-3p mimic vs miR-132-3p mimic + GLRX & 3 & Yes & & & & & Tukey & 0.002 \\
\hline $6 C$, IL-6 & 3 & Yes & One-way ANOVA & $F_{(2,6)}=36.31$ & 0.0004 & Treatment & & \\
\hline $6 C$, IL-6, mimic NC vs miR-132-3p mimic & 3 & Yes & & & & & Tukey & 0.0013 \\
\hline $6 C$, IL-6, miR-132-3p mimic vs miR-132-3p mimic + GLRX & 3 & Yes & & & & & Tukey & 0.0005 \\
\hline $6 D$, TNF- $\alpha$ & 3 & Yes & One-way ANOVA & $F_{(2,6)}=49.81$ & 0.0002 & Treatment & & \\
\hline $6 D$, TNF- $\alpha$, mimic NC vs miR-132-3p mimic & 3 & Yes & & & & & Tukey & 0.0002 \\
\hline $6 D$, TNF- $\alpha$, miR-132-3p mimic vs miR-132-3p mimic + GLRX & 3 & Yes & & & & & Tukey & 0.0011 \\
\hline $6 D, \mathrm{IL}-1 \beta$ & 3 & Yes & One-way ANOVA & $F_{(2,6)}=131.6$ & $<0.0001$ & Treatment & & \\
\hline $6 D, \mathrm{IL}-1 \beta$, mimic NC vs miR-132-3p mimic & 3 & Yes & & & & & Tukey & $<0.0001$ \\
\hline $6 D, \mathrm{IL}-1 \beta, \mathrm{miR}-132-3 \mathrm{p}$ mimic vs miR-132-3p mimic $+\mathrm{GLRX}$ & 3 & Yes & & & & & Tukey & $<0.0001$ \\
\hline $6 D$, IL-6 & 3 & Yes & One-way ANOVA & $F_{(2,6)}=65.52$ & $<0.0001$ & Treatment & & \\
\hline $6 D$, IL-6, mimic NC vs miR-132-3p mimic & 3 & Yes & & & & & Tukey & 0.0001 \\
\hline $6 D$, IL-6, miR-132-3p mimic vs miR-132-3p mimic + GLRX & 3 & Yes & & & & & Tukey & 0.003 \\
\hline $6 E$ & 3 & Yes & One-way ANOVA & $F_{(2,6)}=8.975$ & 0.0157 & Treatment & & \\
\hline $6 E$, mimic NC vs miR-132-3p mimic & 3 & Yes & & & & & Tukey & 0.0199 \\
\hline $6 E$, miR-132-3p mimic vs miR-132-3p mimic + GLRX & 3 & Yes & & & & & Tukey & 0.0312 \\
\hline $6 F$ & 3 & Yes & One-way ANOVA & $F_{(2,6)}=15.91$ & 0.004 & Treatment & & \\
\hline $6 F$, mimic NC vs miR-132-3p mimic & 3 & Yes & & & & & Tukey & 0.0047 \\
\hline $6 F$, miR-132-3p mimic vs miR-132-3p mimic + GLRX & 3 & Yes & & & & & Tukey & 0.0103 \\
\hline $7 A$ & 6 & Yes & One-way ANOVA & $F_{(3,20)}=65.02$ & $<0.0001$ & Treatment & & \\
\hline $7 A$, saline vs MPTP & 6 & Yes & & & & & Tukey & $<0.0001$ \\
\hline $7 A, \mathrm{MPTP}+$ antagomir NC vs MPTP $+\mathrm{miR}-132-3 p$ antagomir & 6 & Yes & & & & & Tukey & 0.001 \\
\hline $7 B$ & 6 & Yes & One-way ANOVA & $F_{(3,20)}=35.37$ & $<0.0001$ & Treatment & & \\
\hline $7 B$, saline vs MPTP & 6 & Yes & & & & & Tukey & $<0.0001$ \\
\hline $7 B, \mathrm{MPTP}+$ antagomir NC vs MPTP $+\mathrm{miR}-132-3 p$ antagomir & 6 & Yes & & & & & Tukey & 0.001 \\
\hline
\end{tabular}

cell supernatant was abandoned before the sediments were washed for six times. The magnetic bead-antibody complex was resuspended in $150 \mu \mathrm{l}$ of Proteinase $\mathrm{K}$ buffer and incubated at $55^{\circ} \mathrm{C}$ for $30 \mathrm{~min}$. Then, the samples were put in the magnetic separation rack to remove the supernatant. The gene expression was analyzed by qRT-PCR after RNA extraction.

\section{Dual-luciferase reporter assay}

The binding site of miR-132-3p and GLRX was predicted by the online prediction software StarBase (http://starbase.sysu.edu.cn/). The mutated type and wild-type sequences in the binding sites were designed and cloned into pGL3-Promoter luciferase plasmid (Promega), namely mut-GLRX and wt-GLRX. Then, mut-GLRX or wt-GLRX was cotransfected with miR132-3p mimic or miR-132-3p inhibitor, respectively, into HEK-239T cells or pRL-TK (Promega). After that, Renilla luciferase activity and Firefly luciferase activity were determined by dual-luciferase reporter gene assay kit (Promega). Renilla luciferase activity was deemed as the internal control, and the ratio between the activities of Firefly luciferase and Renilla luciferase was calculated as the relative activity.

\section{PD mouse model}

Six-month-old male C57BL/6J mice $(n=24)$ were purchased from the Shanghai SLAC Laboratory Animal Co, Ltd. All animal handling and experimental procedures were approved by the Animal Care and Use Committee of Hunan Provincial People's Hospital (No. 202004). Mice were allowed food and water ad libitum and housed in rooms maintained at $24 \pm 1^{\circ} \mathrm{C}$ and $60-80 \%$ humidity using a 12-h dark cycle. The following experiments were conducted after one week of feeding. The study consisted of four groups of six mice each (random grouping by an Excel random number generator): the 1-methyl-4-phenyl1,2,3,6-tetrahydropyridine (MPTP), saline, miR-132-3p antagomir and antagomir NC groups. Mice in the MPTP group were intraperitoneally injected with $30 \mathrm{mg} / \mathrm{kg}$ MPTP 
(Sigma-Aldrich) every day for five consecutive days (Hu et al., 2019), and the mice of the Saline group were intraperitoneally injected with the same amount of Saline every day for five consecutive days. Mice in the MPTP $+m i R-$ $132-3 p$ antagomir group or MPTP + antagomir NC group were injected with MPTP the next day after stereotactic injection of miR-132-3p antagomir or antagomir NC (20 $\mathrm{nm}$, total volume of $5 \mu \mathrm{l}$, GenePharma) into targeted brain areas of mice.

\section{Stereotactic injection}

After anesthesia of mice with ketamine $(100 \mathrm{mg} / \mathrm{kg})$ and xylazine $(10 \mathrm{mg} / \mathrm{kg})$ by intraperitoneal injection, the head of mouse was fixed to expose the skull. The intracerebral injection was performed on the following coordinates: $-2.8 \mathrm{~mm}$ anteroposterior, $-1.2 \mathrm{~mm}$ mediolateral, and $-4.3 \mathrm{~mm}$ dorsoventral. Five microliters of $m i R-132-3 p$ antagomir suspension or antagomir NC suspension was injected into SNc by using a $10-\mu \mathrm{l}$ stereotactic catheter $(1 \mu \mathrm{l} / 5 \mathrm{~min})$. The needle remained in place for $5 \mathrm{~min}$ after complete injection then slowly removed. The mice were placed on a pad until recovery from the anesthesia. The healthy conditions of mice were monitored on the following $5 \mathrm{~d}$, during which mice were subjected to acesodyne and local disinfection. The activity of mice after injection was recorded. No mouse was died during the whole experiments.

\section{Behavioral tests}

One week after establishment of PD mouse models, the behavioral tests were commenced. Motor coordination ability of experimental animals was investigated with the rotarod test. Before the experiments, animals were placed on rotating lanes for $5 \mathrm{~min}$. Then, mice were trained for $2 \mathrm{~min}$ at a fixed speed of $4 \mathrm{rpm}$. After training, the rotational speed was accelerated uniformly from $4 \mathrm{rpm}$ to $40 \mathrm{rpm}$ within $60 \mathrm{~s}$. The time of mice falling off the rotating rod was recorded. The open field test was conducted to evaluate the autonomous and exploratory behaviors of experimental animals in novel environments. Mice were individually placed into the center of an open field box $(38 \times 38 \mathrm{~cm})$ in a noise and light-controlled room. The spontaneous locomotor activities (central-area distance and whole-area distance) of each mouse were recorded and analyzed. The relative time of mouse falling from the rotating rod and the relative distance of mouse staying in the open field were recorded. Relative time and relative distance are the ratio of time or distance of experimental mouse/control mouse.

\section{Brain tissue collection}

Approximately $24 \mathrm{~h}$ after behavior test, ketamine $(100 \mathrm{mg} / \mathrm{kg})$ and xylaafine $(10 \mathrm{mg} / \mathrm{kg})$ were given to mouse for anesthesia through intraperitoneal injection. The heart was exposed and mouse $(n=6)$ in each group was perfused with $200 \mathrm{ml}$ of normal saline through ventriculus sinister. The skull was opened and the brain tissues were collected and isolated. Part of the brain tissues was stored at $-80^{\circ} \mathrm{C}$ refrigerator for $\mathrm{qRT}-\mathrm{PCR}$ and the rest brain tissues were fixed in $4 \%$ triformol for $48 \mathrm{~h}$ for fluorescence in situ hybridization (FISH), immunofluorescence and immunohistochemistry.

\section{FISH}

The 4- $\mu \mathrm{m}$ paraffin sections were de-paraffinized with xylene and gradient alcohol (xylene soak for $10 \mathrm{~min}$, refresh xylene for another $10 \mathrm{~min}, 50 \%$ xylene soaking for $10 \mathrm{~min}$, absolute ethanol for $5 \mathrm{~min}$, refresh absolute ethanol for another $5 \mathrm{~min}, 95 \%$ ethanol for $5 \mathrm{~min}, 90 \%$ ethanol for $5 \mathrm{~min}, 80 \%$ ethanol for $5 \mathrm{~min}$, and $70 \%$ ethanol for $5 \mathrm{~min}$ ) before PBS wash. Sections were digested with $37^{\circ} \mathrm{C}$ protease $\mathrm{K}$ for $15 \mathrm{~min}$, washed with PBS for 2 $\times 5 \mathrm{~min}$, prehybridized at $55^{\circ} \mathrm{C}$ constant temperature, and incubated with digoxigenin-labeled (Exiqon) miR$132-3 p$ probes overnight at incubator with $55^{\circ} \mathrm{C}$ constant temperature. Then, sections were subsequently washed with $5 \times$ SSC buffer, $1 \times$ SSC, and $0.2 \times$ SSC buffer for $2 \times 5 \mathrm{~min}$ at $55^{\circ} \mathrm{C}$, followed by $5 \mathrm{~min}$ of wash with $0.2 \times$ SSC buffer at room temperature, $10 \mathrm{~min}$ of inactivation with $0.3 \%$ hydrogen peroxide-methanol solution, and $3 \times 5 \mathrm{~min}$ of PBS wash. After that, sections underwent three times of incubation each for $1 \mathrm{~h}$ : first blocked with normal serum blocking buffer at room temperature, second probed with mouse anti-DIG at room temperature, and then incubated with polymer anti-mouse. After each incubation, sections were washed three times with PBS for $5 \mathrm{~min}$. Sections were stained with DAB for 5-10 min and washed with tap water for $10 \mathrm{~min}$, before $2 \mathrm{~min}$ of hematoxylin counterstaining, hydrochloric ethanol differentiation and 10 min of tap water wash. These sections were sealed with neutral balata for observation under a microscope after dehydration and permeabilization. The ratio of positive cell numbers to total number of cells was calculated. DAPI was used for staining of cell nucleus and Iba1 was used to labeled microglial cells.

\section{Immunofluorescence}

Sections were incubated for $60 \mathrm{~min}$ at $60^{\circ} \mathrm{C}$, dewaxed with xylene and washed with distilled water. Following antigen retrieval with $0.01 \mathrm{~mol} / \mathrm{l}$ sodium citrate, sections were subjected to 10 min of incubation with $3 \% \mathrm{H}_{2} \mathrm{O}_{2}$. Then, sections were washed with PBS for $3 \times 5 \mathrm{~min}$, and inactivated with $5 \%$ normal goat serum for $30 \mathrm{~min}$ at room temperature. Sections were cultured with the primary antibody against tyrosine hydroxylase (ab137869, 1:200, Abcam), Iba1 (ab178846, 1:500, Abcam) or GLRX (ab45953, $1 \mu \mathrm{g} / \mathrm{ml}$, Abcam) overnight at $4^{\circ} \mathrm{C}$, while sections in negative control group contains corresponding antigens and were incubated with PBS. After that, sections were washed three times with PBS and incubated with FITC-labeled secondary antibody (ab6785, 1:1000, Abcam) at room temperature for $1 \mathrm{~h}$. Then, the secondary antibody was removed, and cells were subjected to 5 min of staining with DAPI and $3 \times 5$ min of PBS wash. Before pictures were captured by fluorescence microscope, sections were given glycerophosphoric acid for sealing. Stereolnvestigator (MBF Bioscience) was used for stereological analysis on the total number of $\mathrm{TH}$ 
A

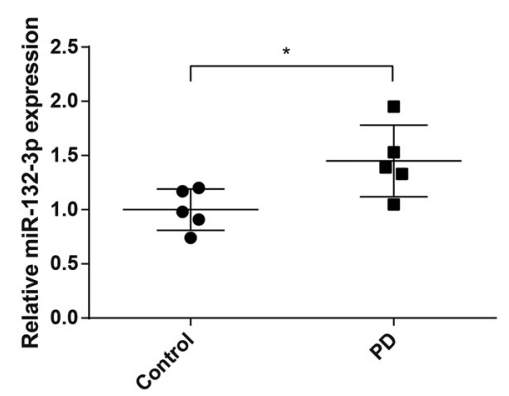

C

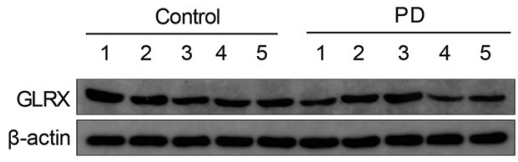

B
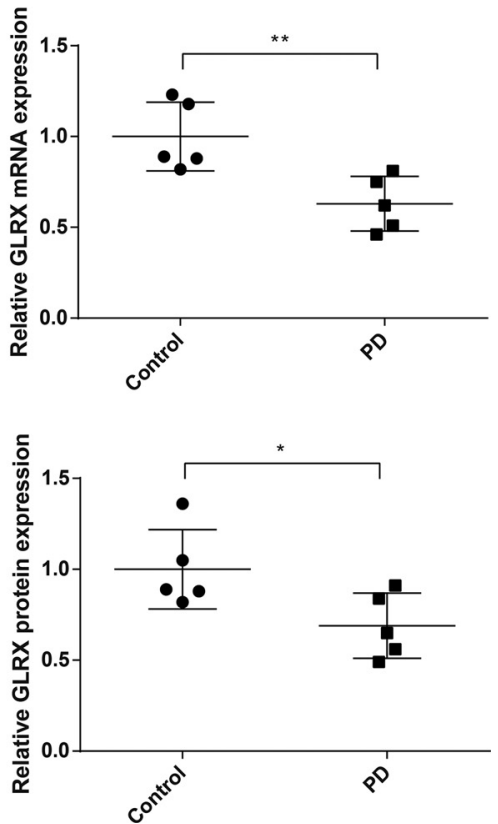

Figure 1. Expressions of miR-132-3p and GLRX in the midbrain tissues of patients with PD. The expression of miR-132-3p in the midbrain tissues of five PD patients and five age-matched controls was detected by qRT-PCR $(\boldsymbol{A})$, and the mRNA and protein expressions of GLRX were measured by qRT-PCR $(\boldsymbol{B})$, and Western blotting $(\boldsymbol{C}) ; N$ (number of participants) $=5,{ }^{\star} p<0.05,{ }^{* \star} p<0.01$, Error bars, standard deviation (SD).

positive neurons and Iba1 positive cells for every sixth coronal section through the midbrain. After the the SN pars compacta with a $4 \times$ objective was delineated, cells were counted under $\times 60$ magnification using ImageJ and following parameters: $8-\mu \mathrm{m}$ height of an optical dissector, $50 \times 50 \mu \mathrm{m}$ counting frame, $100 \times 100 \mu \mathrm{m}$ area of a grid. The error coefficient of $<0.10$ was acceptable. All sections were quantified in a blinded manner.

\section{Immunohistochemistry}

Following $60 \mathrm{~min}$ of bake, sections were dewaxed by $x y-$ lene and washed with distilled water. Before $30 \mathrm{~min}$ of inactivation with normal goat serum at room temperature, sections underwent the following steps: antigen retrieval with $0.01 \mathrm{~mol} /$ I sodium citrate, 10 min of reaction with $3 \% \mathrm{H}_{2} \mathrm{O}_{2}$ and three times of PBS wash for $5 \mathrm{~min}$. The sections were inactivated with $5 \%$ normal goat serum for $30 \mathrm{~min}$ at room temperature. After that, sections were incubated with antibody against GLRX (ab45953, $1 \mu \mathrm{g} / \mathrm{ml}$, Abcam) overnight at $4^{\circ} \mathrm{C}$, while sections in negative control group contains corresponding antigens and were incubated with PBS. Sections were then subjected to three times of PBS wash and $1 \mathrm{~h}$ of incubation with secondary antibody (ab6785, 1:1000, Abcam). DAB was used for color development, and sections were given three times of PBS wash to terminate the color reaction (1-3 min). The nucleus was stained with hematoxylin for $3 \mathrm{~min}$, and sections were dehydrated, permeabilized, and sealed. The percentage of positive cells was counted. Images were analyzed using ImageJ software (version 1.46, National Institutes of Health).

\section{Statistical analysis}

Experiments and statistical analysis were performed by different personnel. Statistical analysis was conducted using GraphPad Prism 7 software, and data are displayed as the mean $\pm \mathrm{SD}$. The normal distribution of data was detected by Kolmogorov-Smirnov test, D'Agostino, Pearson omnibus normality test, or Shapiro-Wilk normality test. All data were complied with normal distribution. The $t$ test was employed for comparisons between two groups. The one-way ANOVA was adopted followed by Tukey's multiple comparison tests for comparisons among multiple groups. $P$ values of significance were those $<0.05$ (Tables 2, 3, 4).

\section{Results}

\section{Highly expressed $m i R-132-3 p$ and lowly expressed GLRX in midbrain tissues of patients with PD}

The expressions of miR-132-3p and GLRX in midbrain tissues of patients with PD and in healthy controls were determined by qRT-PCR and Western blotting. The results of qRT-PCR manifested compared with control group, miR-132-3p in midbrain tissues of patients with PD was increased by $1.45 \pm 0.33$-fold $(p<0.05$; Fig. $1 A)$. Furthermore, analyses of QRT-PCR and Western blotting exhibited that the mRNA and protein expressions of GLRX in the tissues of patients with PD were decreased to respectively $0.63 \pm 0.15$-fold and $0.69 \pm 0.18$-fold $(p<$ 0.05 , vs the control group; Fig. $1 B, C)$. These finding indicated that $m i R-132-3 p$ and GLRX may be implicated in the progression of $\mathrm{PD}$.

\section{Knock-down of $m i R-132-3 p$ inhibits LPS-induced inflammatory response in BV-2 cells}

The BV-2 cells were transfected with miR-132-3p inhibitor or inhibitor NC before $0.1 \mu \mathrm{g} / \mathrm{ml}$ LPS or PBS treatment to explore the effect of miR-132-3p on LPS-induced 
A

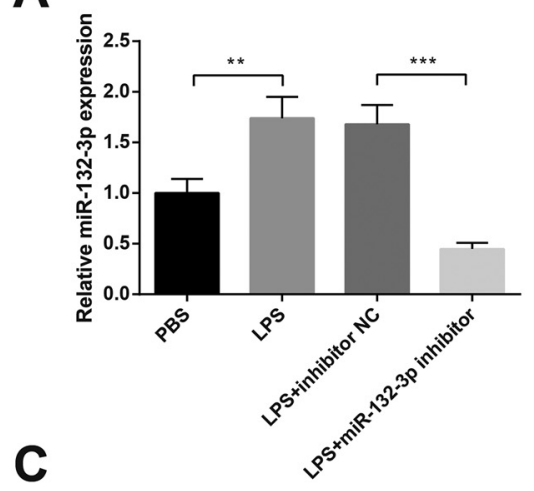

B

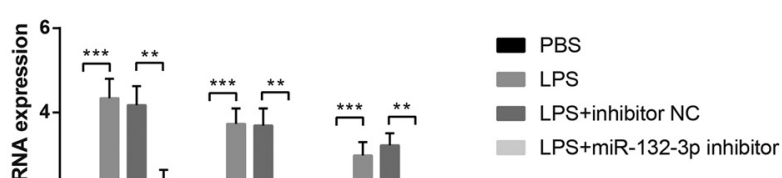

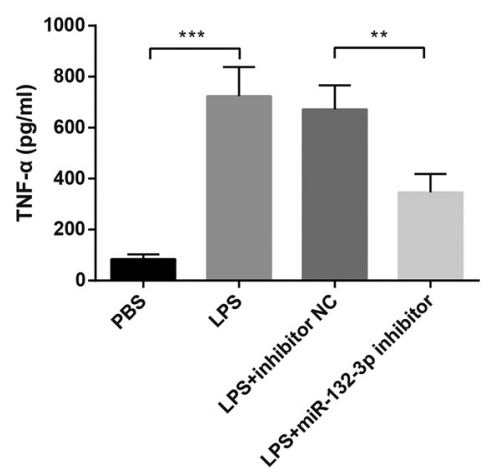
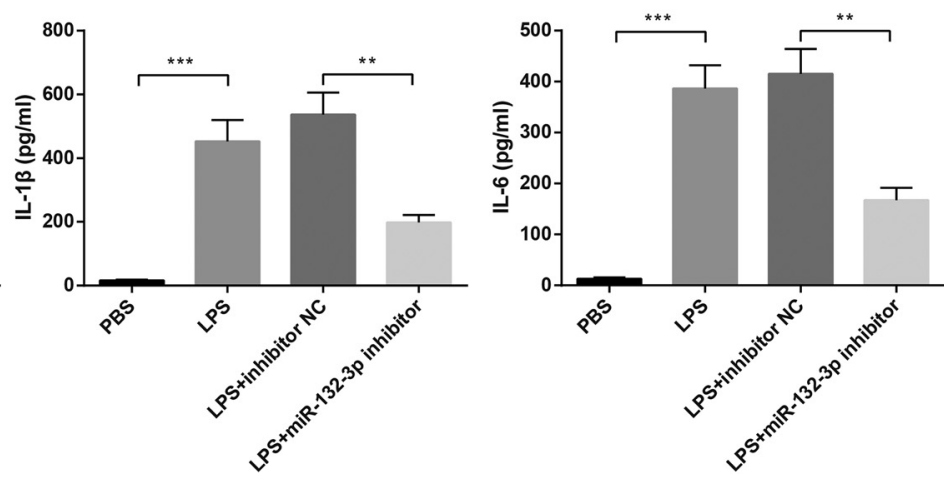

Figure 2. LPS-induced inflammatory response in BV-2 microglial cells can be attenuated by miR-132-3p knock-down. Following transfection with miR-132-3p inhibitor or inhibitor NC, BV-2 cells were treated with $0.1 \mu \mathrm{g} / \mathrm{ml}$ LPS or PBS for $24 \mathrm{~h}$. qRT-PCR was used to detect the expression of miR-132-3p in BV-2 cells $(\boldsymbol{A})$. The mRNA expressions of inflammatory cytokines TNF- $\alpha$, IL-1 $\beta$, and IL-6 were analyzed by qRT-PCR $(\boldsymbol{B})$, and the contents of TNF- $\alpha$, IL- $1 \beta$, and IL- 6 in the supernatant of BV- 2 cells were determined by ELISA (C); $N$ (number of independent cell culture preparations) $=3,{ }^{\star *} p<0.01,{ }^{\star \star *} p<0.001$, Error bars, standard deviation (SD).

inflammatory response in BV-2 cells. Results of qRT-PCR presented that LPS treatment elevated miR-132-3p expression in BV-2 cells by $1.74 \pm 0.21$-fold $(p<0.01$, vs the PBS group), while transfection with miR-132-3p inhibitor suppressed the level of miR-132-3p $(p<0.001$, LPS + miR-132-3p inhibitor vs the LPS + inhibitor NC group: $0.45 \pm 0.06$-fold vs $1.68 \pm 0.19$-fold; Fig. $2 A$ ). Additionally, the enhanced expressions of inflammatory cytokines IL-6 (2.98 \pm 0.32 -fold), TNF- $\alpha \quad(4.34 \pm 0.46$-fold $)$ and IL- $1 \beta$ $(3.73 \pm 0.37$-fold) in BV-2 cells were occurred in response to LPS induction ( $p<0.001$, vs the PBS group), whereas these levels were somewhat attenuated in cells transfected with miR-132-3p inhibitor $(p<0.01$, LPS + miR-132-3p inhibitor vs the LPS+inhibitor NC group: TNF- $\alpha$ : $2.38 \pm 0.26$-fold vs $4.18 \pm 0.45$-fold; IL-1 $\beta: 1.69 \pm 0.18$-fold vs $3.68 \pm 0.41$-fold; IL-6: $1.84 \pm 0.21$-fold vs $3.22 \pm 0.29$-fold; Fig. $2 B$ ). The contents of inflammatory cytokines in the supernatant of BV-2 cells were determined by ELISA, and the results showed that the rises in contents of IL- $1 \beta \quad(453.29 \pm 66.47$ vs $16.74 \pm 2.58 \mathrm{pg} / \mathrm{ml}), \mathrm{IL}-6$ (386.47 \pm 45.79 vs $13.27 \pm 2.18 \mathrm{pg} /$ $\mathrm{ml})$ and TNF- $\alpha(734.48 \pm 114.37$ vs $84.56 \pm 18.24 \mathrm{pg} / \mathrm{ml})$ in the LPS group ( $p<0.001$, vs the PBS group). Compared with LPS + inhibitor NC group, contents of IL-1 $\beta$ (198.37 \pm 23.51 vs $537.28 \pm 68.34 \mathrm{pg} / \mathrm{ml}), \mathrm{IL}-6 \quad(167.28 \pm 24.35$ vs $415.27 \pm 48.61 \mathrm{pg} / \mathrm{ml})$, and TNF- $\alpha(346.57 \pm 72.44$ vs $672.35 \pm 94.27 \mathrm{pg} / \mathrm{ml}$ ) in the LPS $+m i R-132-3 p$ inhibitor group were suppressed ( $p<0.01$; Fig. 2C). The above findings suggested that suppression on miR-132-3p may repress LPS-induced inflammatory response in BV-2 microglial cells.

\section{miR-132-3p overexpression enhances inflammatory response in BV-2 cells}

Based on the previous experimental results, downregulation of miR-132-3p can inhibit LPS-induced activation and inflammation of microglial cells. However, whether upregulation of miR-132-3p can induce microglial activation and inflammation is still unknown. Toward this end, BV-2 cells were transfected with miR-132-3p mimic or mimic NC. We found that overexpression of $m i R-132-3 p$ in miR-132-3p mimic group had increased the levels of miR-132-3p by $6.84 \pm 0.53$-fold ( $p<0.001$; Fig. $3 A$ ). Additionally, miR-132-3p mimic group had elevated mRNA expressions of TNF- $\alpha(1.89 \pm 0.19$-fold vs $0.92 \pm 0.12$ fold), IL- $1 \beta$ (2.15 \pm 0.22 -fold vs $1.06 \pm 0.15$-fold), IL-6 (1.75 \pm 0.16 -fold vs $1.13 \pm 0.11$-fold) when compared with mimic NC group ( $p<0.01$; Fig. 3B). ELISA showed that TNF- $\alpha \quad(247.63 \pm 29.14$ vs $82.78 \pm 15.69 \mathrm{pg} / \mathrm{ml}), \quad \mathrm{IL}-1 \beta$ $(172.59 \pm 19.37$ vs $15.94 \pm 1.83 \mathrm{pg} / \mathrm{ml}), \quad$ IL-6 $(134.76 \pm$ 16.72 vs $15.36 \pm 1.87 \mathrm{pg} / \mathrm{ml})$ in the supernatant of BV-2 cells was also elevated in miR-132-3p mimic group in 
A

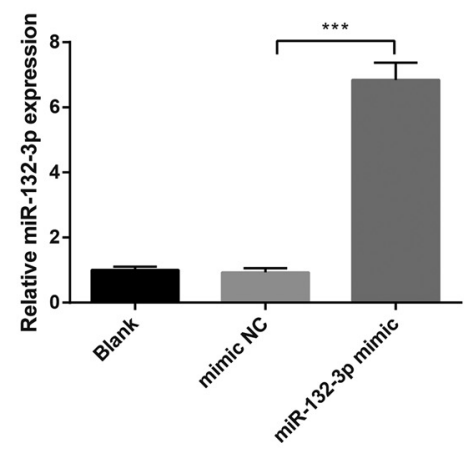

B

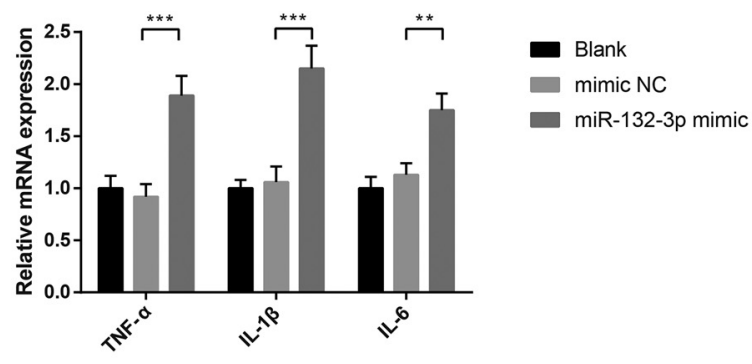

C

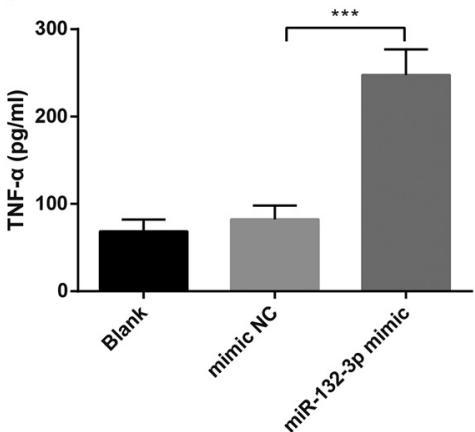

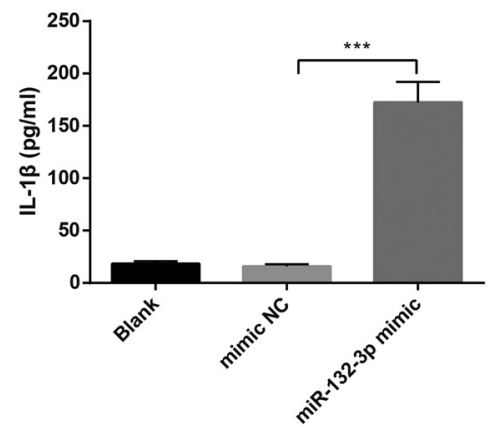

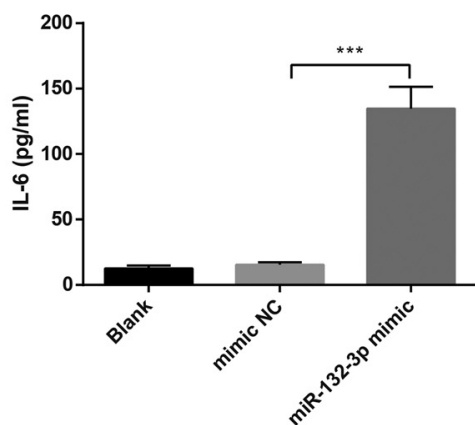

Figure 3. Overexpression of miR-132-3p promotes the release of proinflammatory cytokines in BV-2 microglial cells. After BV-2 cells transfected with miR-132-3p mimic or mimic NC, the mRNA expression of miR-132-3p in BV-2 cells $(\boldsymbol{A})$ and the mRNA expressions of inflammatory cytokines TNF- $\alpha$, IL-1 $\beta$, and IL- 6 in BV-2 cells were examined by qRT-PCR (B). Then, ELISA was utilized to assess the contents of TNF- $\alpha$, IL-1 $\beta$, and IL-6 in the supernatant of BV-2 cells (C); $N$ (number of independent cell culture preparations) $=3,{ }^{\star \star} p<0.01,{ }^{\star \star \star} p<0.001$, Error bars, standard deviation (SD).

contrast to mimic NC group $(p<0.001$; Fig. $3 C)$. The results demonstrated that overexpression of miR-132-3p may induce the activation and inflammatory response of microglial cells.

\section{Activated microglial cells by miR-132-3p cause neuronal injury}

To investigate the role of microglial activation in neuronal injury, SH-SY5Y cells were cultured in conditioned medium of BV-2 cells that were transfected with inhibitor NC or miR-132-3p inhibitor and stimulated with LPS. The findings of CCK-8 assay addressed that the viability of SH-SY5Y cells in the LPS group was decreased to $43.86 \pm 3.86 \%$ compared with PBS group $(p<0.001)$, while LPS $+m i R-132-3 p$ inhibitor group possessed higher cell viability than that in the LPS + inhibitor NC group $(p<$ $0.01,71.61 \pm 6.23 \%$ vs $50.27 \pm 4.14 \%$; Fig. $4 A$ ). Flow cytometry assessed that LPS stimulation heightened the apoptotic rate of SH-SY5Y cells $(p<0.001,37.84 \pm 2.73 \%$ vs $8.67 \pm 1.23 \%$; Fig. $4 B$ ), but treatment with miR-132-3p inhibitor lowered cell apoptotic rate $(p<0.001,19.34 \pm$ $2.27 \%$ vs $40.25 \pm 3.16 \%$; Fig. $4 B$ ).

Meanwhile, the effect of miR-132-3p overexpression on neuronal injury was explored. Accordingly, SH-SY5Y cells were cultured in conditioned medium, in which BV-2 cells were transfected with mimic NC or miR-132-3p mimic. We discovered that upregulation of miR-132-3p reinforced cell apoptotic rate $(16.83 \pm 2.37 \%$ vs $8.25 \pm 1.43 \%)$ and diminished the viability of SH-SY5Y cells $(71.35 \pm 9.61 \%$ vs $93.15 \pm$ 8.63\%; Fig. $4 C, D, p<0.05$ ). Collectively, the activated microglial cells by miR-132-3p may lead to neuronal injury.

\section{MiR-132-3p is an upstream regulatory factor of GLRX}

After knock-down or overexpression of $m i R-132-3 p$ in BV-2 cells, the mRNA and protein expressions of GLRX were evaluated by qRT-PCR and Western blotting. We found that enhanced GLRX expression in miR-132-3p inhibitor group when compared with inhibitor NC group $(p<0.05$, mRNA: $1.76 \pm 0.21$-fold vs $1.09 \pm 0.12$-fold; protein: $1.58 \pm 0.23$-fold vs $0.95 \pm 0.13$-fold; Fig. $5 A, B$ ), while miR-132-3p mimic group had repressed level of GLRX compared with mimic NC group $(p<0.05$, mRNA: $0.54 \pm 0.07$-fold vs $0.94 \pm 0.11$-fold; protein: $0.64 \pm 0.11$ fold vs $0.96 \pm 0.16$-fold; Fig. $5 A, B$ ). Subsequently, the RIP experiment was applied to verify the relationship of $m i R$ $132-3 p$ to GLRX mRNA, and the results manifested that compared with IgG antibody group, substantial GLRX mRNA can be recruited in Ago2 complex (2.15 $\pm 0.24-$ fold vs $0.12 \pm 0.03$-fold; Fig. $5 C, p<0.001$ ). After LPS treatment, increased GLRX mRNA was recruited in Ago2 complex $(2.45 \pm 0.36$-fold vs $1.68 \pm 0.23$-fold; Fig. $5 D$, $p<0.05)$. Considering the directing binding of miR-132$3 p$ with GLRX mRNA cannot be proved by RIP as the regulation of other miRNAs or target genes cannot be 
A

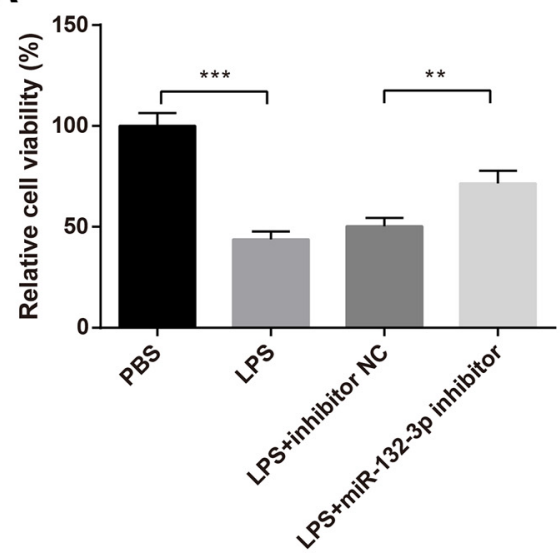

C

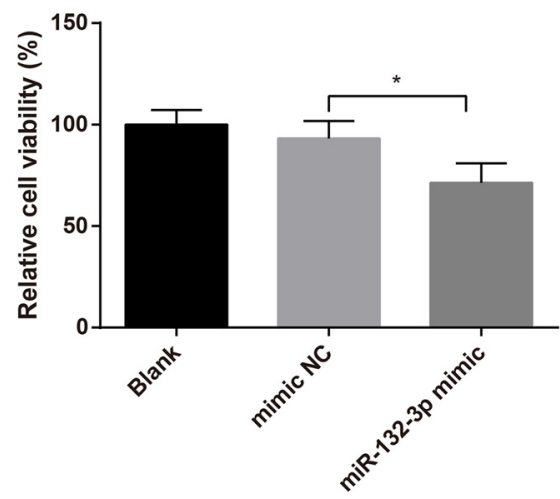

B
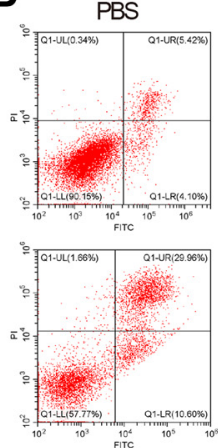

LPS+inhibitor NC
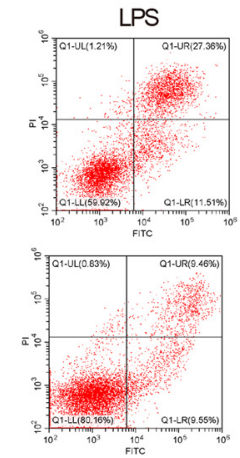

LPS+miR-132-3p inhibitor
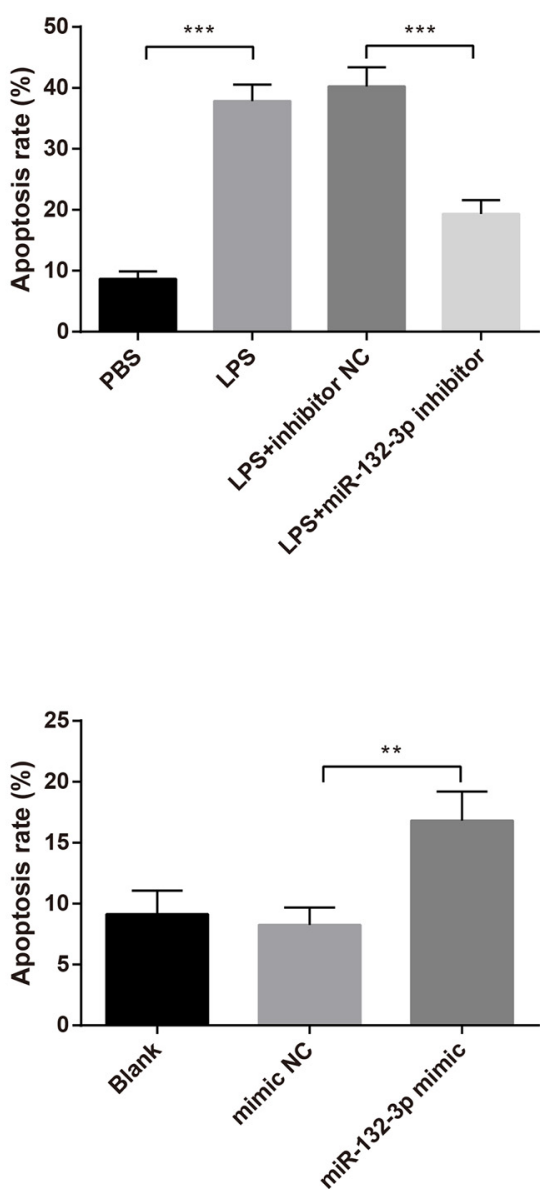

Figure 4. Effect of miR-132-3p induced microglial activation on neuronal injury. The SH-SY5Y cells were cultured in the conditioned medium of BV-2 cells that were transfected with inhibitor NC or miR-132-3p inhibitor and stimulated with LPS. Then, CCK-8 assay was used to detect the viability of SH-SY5Y cells $(\boldsymbol{A})$ and flow cytometry to determine the apoptotic rate $(\boldsymbol{B})$. Additionally, SH-SY5Y cells were cultured in the conditioned medium of BV-2 cells that transfected with miR-132-3p mimic or mimic NC. The viability of SH-SY5Y cells was assessed by CCK-8 assay $(\boldsymbol{C})$ and the apoptotic rate of SH-SY5Y cells was measured by flow cytometry $(\boldsymbol{D}) ;$ (number of independent cell culture preparations) $=3,{ }^{*} p<0.05,{ }^{* *} p<0.01$, ${ }^{* * *} p<0.001$, Error bars, standard deviation (SD).

excluded, we then applied dual luciferase reporter gene assay to verify the binding. The binding site of $m i R-132-3 p$ to the $3^{\prime}$-UTR on GLRX mRNA was predicted by StarBase (Fig. $5 E$ ). The above result was verified by dual-luciferase reporter assay, which displayed that HEK293T cells cotransfected with wt-GLRX and miR-132-3p mimic had decreased luciferase activity by $62.33 \pm 8.17 \%$ than cells cotransfected with wt-GLRX and mimic NC $(p<0.01)$. However, the relative luciferase activity in cells cotransfected with mut-GLRX and miR-132-3p mimic was not statistically different from cells cotransfected with mut-GLRX and mimic NC $(p>0.05$, $108.67 \pm 13.52 \%$ vs $93.16 \pm 11.47 \%$; Fig. $5 F$ ). The above results indicated that miR-132-3p may target GLRX and negatively regulate expression of GLRX.

\section{GLRX mitigates neuronal injury and inhibits activation of microglial cells induced by $\mathbf{m i R}-132-3 p$}

To explore whether miR-132-3p promotes microglial inflammation and neuronal injury through mediating
GLRX, BV-2 cells were transfected miR-132-3p mimic or cotransfected miR-132-3p mimic and plasmid overexpressing GLRX. Results of qRT-PCR and Western blotting highlighted that transfection with miR-132-3p mimic lead to suppressed mRNA and protein expression levels of GLRX by $0.58 \pm 0.08$-fold and $0.64 \pm 0.11$-fold, whereas the coeffect of miR-132-3p mimic and plasmid overexpressing GLRX increased the expression of GLRX compared with transfection with miR-132-3p mimic alone ( $p<0.05$, mRNA: $2.46 \pm 0.31$-fold vs $0.58 \pm 0.08$-fold; protein: $1.85 \pm 0.28$-fold vs $0.64 \pm 0.11$-fold; Fig. $6 A, B$ ). Furthermore, the detections on mRNA expressions and contents of inflammatory cytokines revealed that cells in the miR-132-3p mimic + GLRX group had lower mRNA expression levels and contents of TNF- $\alpha(1.23 \pm 0.14$-fold vs $2.16 \pm 0.22$-fold), IL-6 (0.87 \pm 0.11 -fold vs $1.68 \pm 0.18)$, and IL-1 $\beta$ (1.26 \pm 0.12 -fold vs $1.94 \pm 0.18$-fold $)$ than in the $m i R-$ $132-3 p$ mimic group ( $p<0.01$; Fig. $6 C$ ). ELISA detection showed that the contents of TNF- $\alpha$ (115.86 \pm 12.43 vs $218.37 \pm 26.49 \mathrm{pg} / \mathrm{ml}), \mathrm{IL}-1 \beta$ (49.37 \pm 6.68 vs $159.38 \pm$ 
A

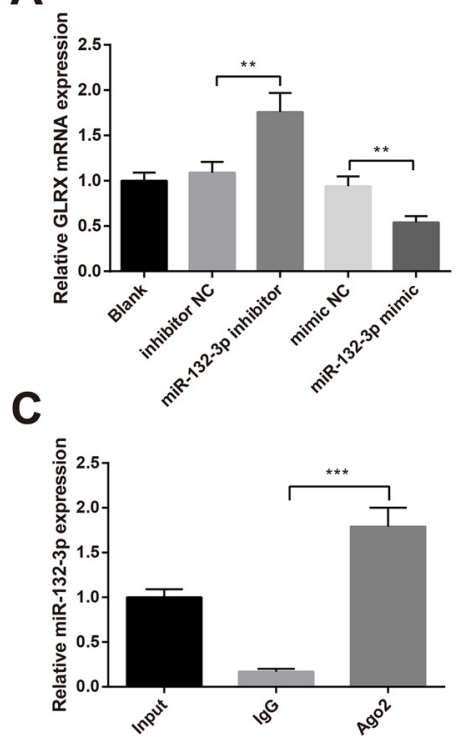

E

wt-GLRX: 5' uccUCAGUGCUAAAAACUGUUg 3' miR-132-3p: 3 | | || | | | || | || mut-GLRX: 5' uccUCAGUGCUAAAAUGACAAg 3'

B

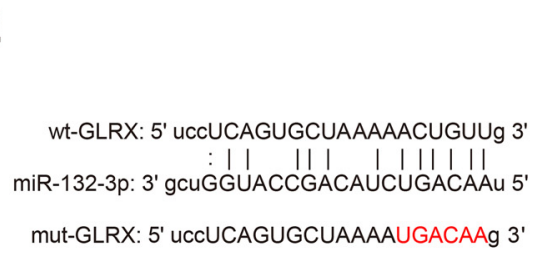

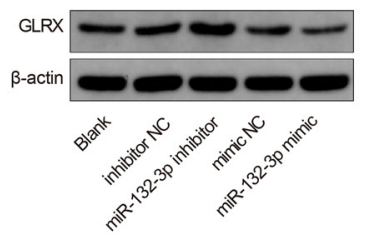
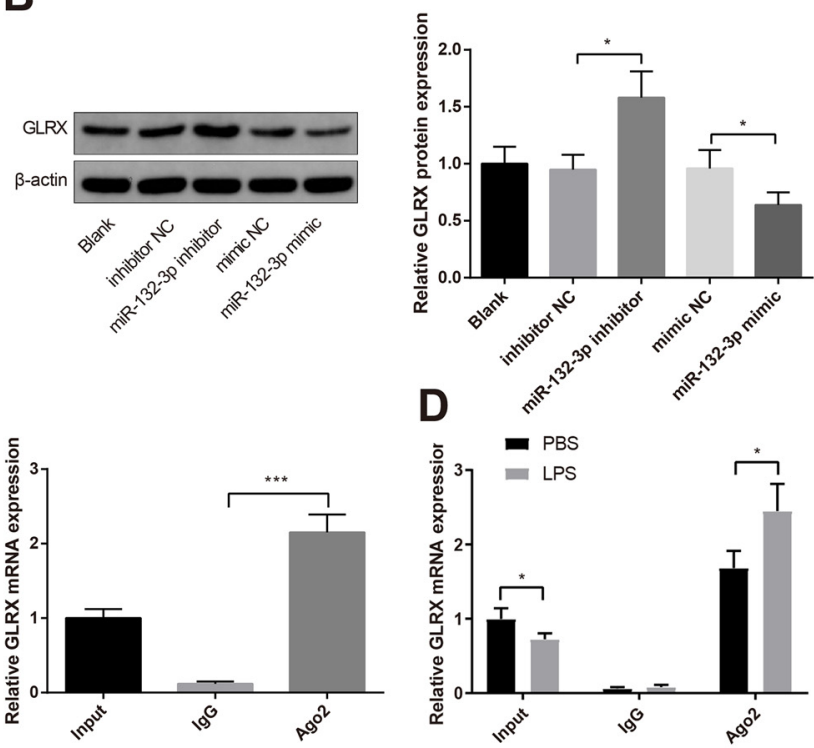

$\mathbf{F}$

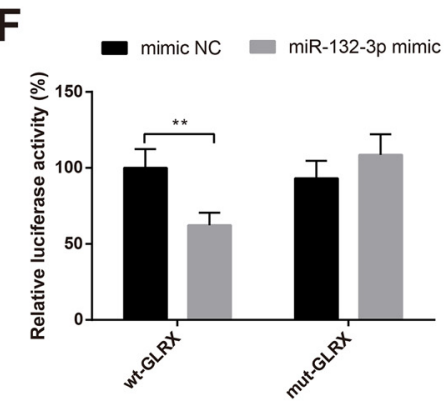

Figure 5. MiR-132-3p negatively mediates GLRX. qRT-PCR $(\boldsymbol{A})$ and Western blotting $(\boldsymbol{B})$ were used to detect the mRNA and protein expressions of GLRX after miR-132-3p knock-down or overexpression in BV-2 cells. RIP experiment was applied to verify the binding of $m i R-132-3 p$ to GLRX mRNA (C). After LPS or PBS treatment, RIP was applied to detect the GLRX mRNA expression in Ago2 complex $(\boldsymbol{D})$. The binding site of miR-132-3p to the 3'-UTR of GLRX mRNA was predicted by StarBase (E). Dual-luciferase reporter assay was utilized to verify the binding relationship between miR-132-3p and GLRX $(\boldsymbol{F}) ; N$ (number of independent cell culture preparations) $=3,{ }^{*} p<0.05,{ }^{* \star} p<0.01,{ }^{* \star *} p<0.001$, Error bars, standard deviation (SD).

$18.32 \mathrm{pg} / \mathrm{ml}), \mathrm{IL}-6$ (31.27 $\pm 5.39 \mathrm{vs} 124.38 \pm 21.15 \mathrm{pg} / \mathrm{ml})$ in the supernatant of BV-2 cells were suppressed in miR132-3p mimic + GLRX group when compared with miR-132$3 p$ mimic group $(p<0.01$; Fig. $6 D)$. Then, SH-SY5Y cells were grown in the conditioned medium of BV-2 cells. The findings of CCK- 8 assay and flow cytometry described the increase in cell viability $(97.39 \pm 7.65 \%$ vs $73.62 \pm 8.51 \%$; Fig. $6 E, p<0.05)$ and the decrease in cell apoptotic rate $(9.66 \pm 2.16 \%$ vs $17.59 \pm 2.67 \%$; Fig. $6 F, p<0.01)$ in the miR-132-3p mimic + GLRX group compared with the miR$132-3 p$ mimic group. These data suggested that overexpression of GLRX may reverse the effect of miR-132-3p upregulation on microglial activation and neuronal injury.

\section{Suppression of $m i R-132-3 p$ alleviates MPTP-induced neuroinflammation and dopaminergic neurodegeneration in PD mouse models}

Mice were subjected to stereotactic injection of miR$132-3 p$ antagomir and given MPTP by intraperitoneal injection to probe the role of miR-132-3p in neuroinflammation and dopaminergic neuron degeneration of MPTP-induced PD mouse. The results of FISH presented that injection with MPTP elevated miR-132-3p expression in SNc of mice by $196.37 \pm 17.39 \%(p<0.001)$, while the following exposure to MPTP $+m i R-132-3 p$ antagomir repressed the level of miR-132-3p $(p<0.01,125.59 \pm$ $12.67 \%$ vs $179.34 \pm 14.34 \%$; Fig. $7 A$ ). Immunohistochemistry results displayed that there were enhanced expression of GLRX in the MPTP $+m i R-132-3 p$ antagomir group $(p<0.01$, vs the MPTP+antagomir NC group, $87.25 \pm 12.57 \%$ vs $57.16 \pm 6.28 \%)$ and decreased level of GLRX in the MPTP group by $53.47 \pm 6.39 \%$ ( $p<0.001$, vs the saline group; Fig. 7B). FISH and immunofluorescence were applied to detect the expressions of miR-132-3p and GLRX in microglial cells. The results showed that miR132-3p expression of SNc of mice in MPTP group was increased by $2.16 \pm 0.36$-fold, while GLRX expression was suppressed by $0.46 \pm 0.11$-fold when compared 
A
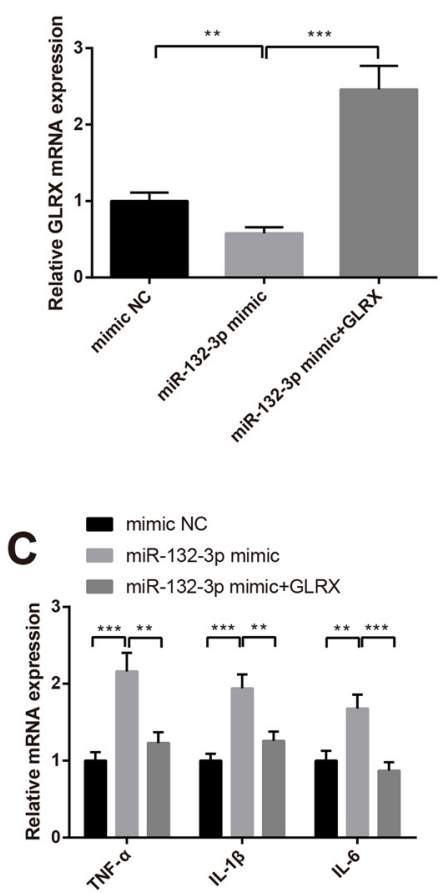

E

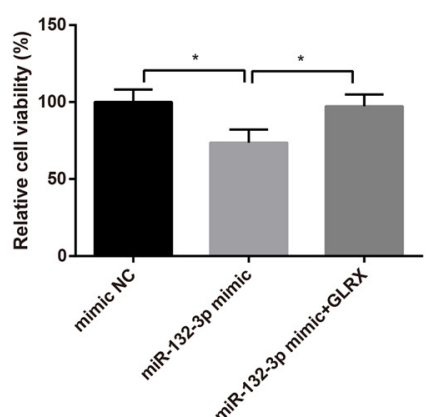

B
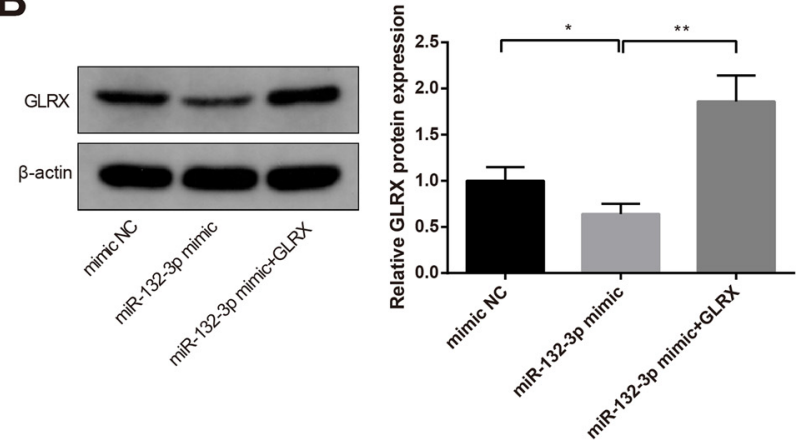

D
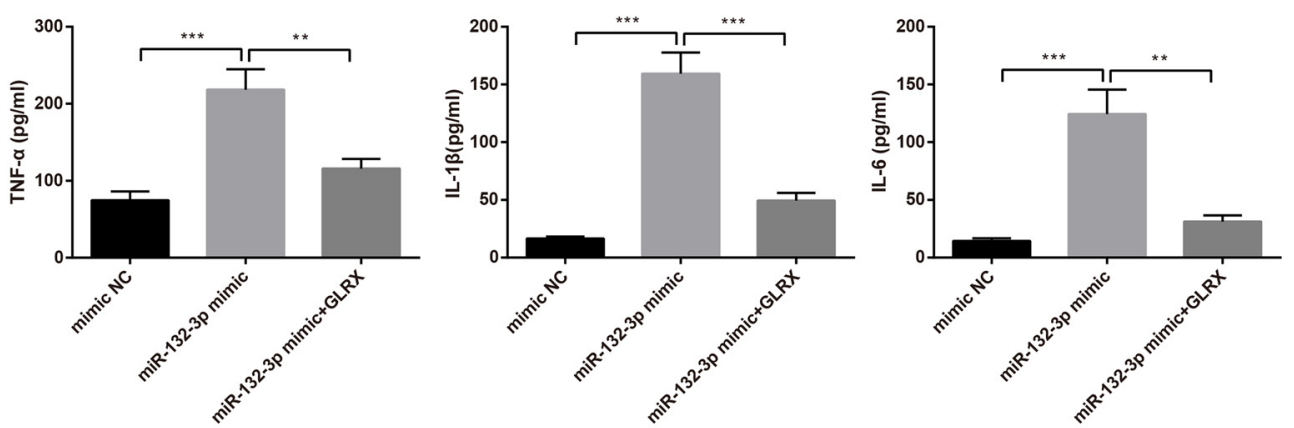

$\mathbf{F}$
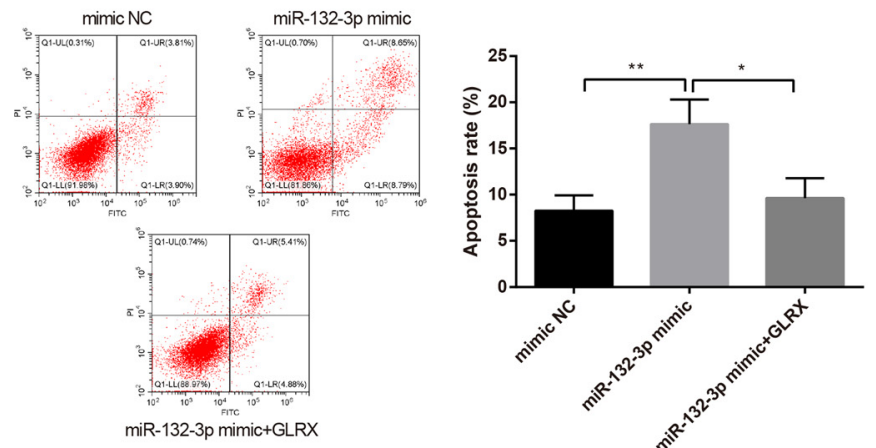

Figure 6. GLRX reverses microglial activation and neuronal injury induced by miR-132-3p. The BV-2 cells were transfected miR132-3p mimic or cotransfected miR-132-3p mimic and GLRX overexpressing plasmid. Then, qRT-PCR $(\boldsymbol{A})$ and Western blotting $(\boldsymbol{B})$ were used to detect the mRNA and protein expressions of GLRX in BV-2 cells. The mRNA expressions of inflammatory cytokines TNF- $\alpha$, IL- $1 \beta$, and IL- 6 in BV-2 cells were examined by qRT-PCR $(\boldsymbol{C})$. Then, ELISA was utilized to assess the contents of TNF- $\alpha$, IL$1 \beta$, and IL-6 in the supernatant of BV-2 cells $(\boldsymbol{D})$. The SH-SY5Y cells were cultured in conditioned medium, in which BV-2 cells were transfected with miR-132-3p mimic or cotransfected miR-132-3p mimic and GLRX overexpressing plasmid. Then, CCK-8 assay was used to detect the viability of SH-SY5Y cells $(\boldsymbol{E})$ and flow cytometry to determine the apoptotic rate $(\boldsymbol{F}) ; N$ (number of independent cell culture preparations) $=3,{ }^{*} p<0.05$, ${ }^{\star \star} p<0.01,{ }^{\star \star \star} p<0.001$, Error bars, standard deviation (SD).

with Saline group. Comparisons between MPTP + miR132-3p antagomir group and MPTP+antagomir NC group demonstrated that miR-132-3p expression was suppressed (miR-132-3p: $1.58 \pm 0.27$-fold vs $2.34 \pm$ 0.38 -fold) and GLRX expression was elevated (GLRX: $0.84 \pm 0.18$-fold vs $0.53 \pm 0.10$-fold) in miR-132-3p antagomir group $(p<0.01$; Fig. $7 C, D)$. Co-location analysis showed miR-132-3p expression in microglial cells accounted for $40 \%$ of miR-132-3p expression in tissues, while the expression of GLRX in microglial cells accounted for $55 \%$ of GLRX expression in tissues (Fig. 7C,D). Additionally, immunofluorescence of Iba1 exhibited that the microglial activation was increased in MPTP group $\left(619.74 \pm 88.90\right.$ vs $\left.174.83 \pm 23.03 \mathrm{cells} / \mathrm{mm}^{2}, p<0.001\right)$ and decreased in MPTP $+m i R-132-3 p$ antagomir group $\left(417.36 \pm 74.78\right.$ vs $595.72 \pm 78.02$ cells $/ \mathrm{mm}^{2}$; Fig. $7 E, p<$ 0.01). Analysis of qRT-PCR showed that MPTP treatment potentiated the mRNA levels of inflammatory cytokines TNF- $\alpha \quad(1.68 \pm 0.29$-fold $), \quad I L-1 \beta \quad(1.79 \pm 0.28$-fold $), \quad I L-6$ $(1.84 \pm 0.32$-fold $)$ in brain tissues of mice $(p<0.01)$, 
A
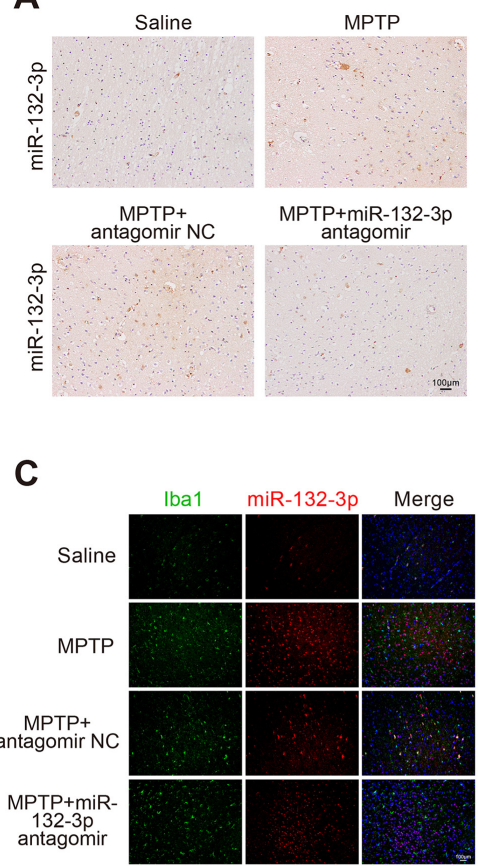

E

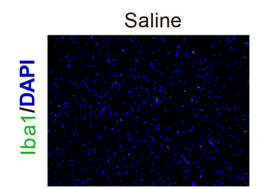

MPTP+
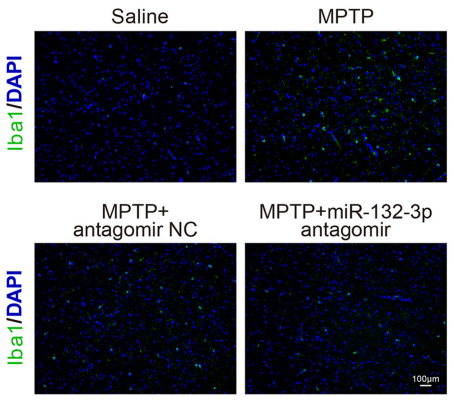
MPTP+miR-132-3p
antagomir

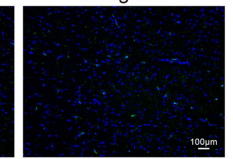

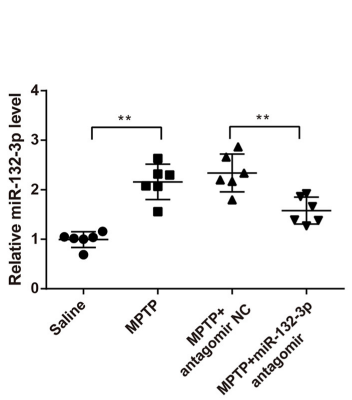

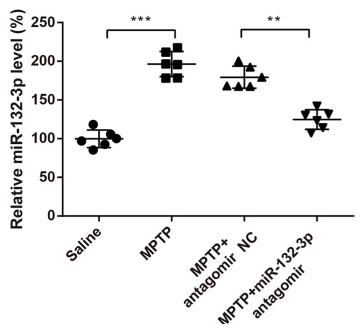

B
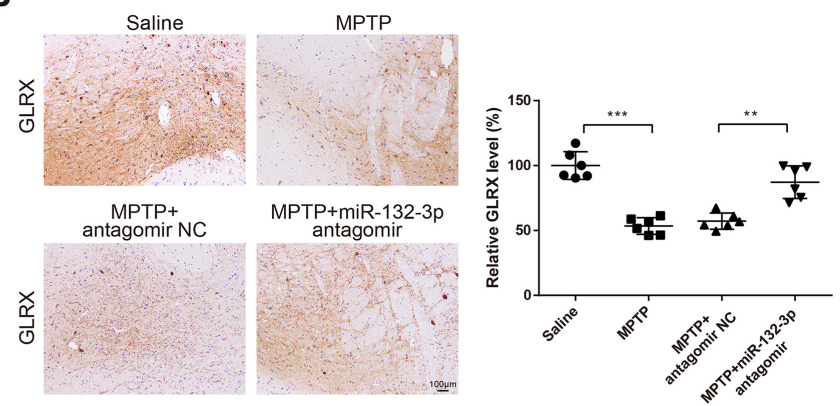

D
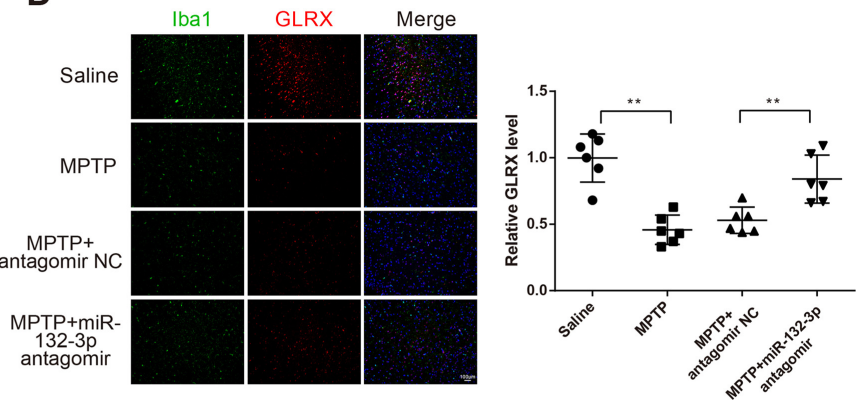

$\mathbf{F}$

- Saline $\triangle \mathrm{MPTP}+$ antagomir NC

- MPTP $\checkmark$ MPTP+miR-132-3p antagomir

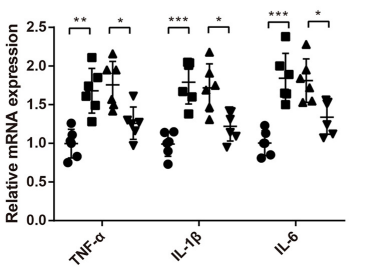

H
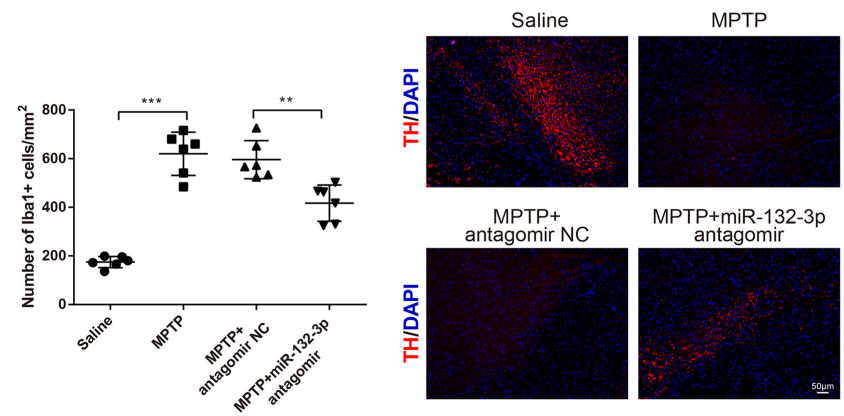

MPTP+miR-132-3p antagomi
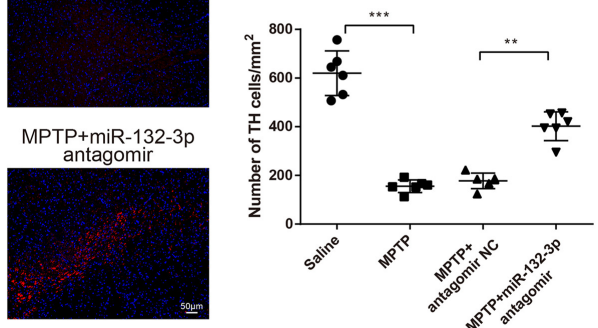

\section{H}
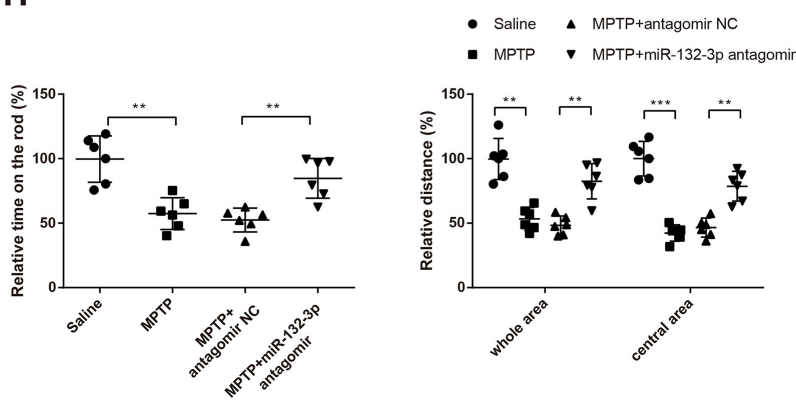

Figure 7. Knock-down of miR-132-3p ameliorates the neuroinflammation and dopaminergic neuron degeneration of PD mouse. Mice were intraperitoneally injected with $30 \mathrm{mg} / \mathrm{kg}$ MPTP to establish PD mouse models. Then, mouse models of PD were given stereotactic injection of miR-132-3p antagomir or antagomir NC. FISH was used to examine the expression of miR-132-3p in SNc of mice $(\boldsymbol{A})$. The expression of GLRX in the SNc of mice was measured by immunohistochemistry (B). FISH was applied to detect the expression of miR-132-3p in microglial cells $(\boldsymbol{C})$. Immunofluorescence was applied to detect the expression of GLRX in microglial cells $(\boldsymbol{D})$; Immunofluorescence of Iba1 was applied to detect the activation of microglial cells $(\boldsymbol{E})$, qRT-PCR to detect the mRNA expressions of TNF- $\alpha, \mathrm{IL}-1 \beta$, and IL- 6 in brain tissues of mouse $(\boldsymbol{F})$, and immunofluorescence of tyrosine hydroxylase to detect the loss of dopaminergic neurons in the SNc of mice (G). The motor ability of mice was assessed after rotarod test and open field test $(\boldsymbol{H}) ; N$ (number of animals) $=6,{ }^{\star} p<0.05,{ }^{* *} p<0.01,{ }^{* \star *} p<0.001$, Error bars, standard deviation (SD). 
whereas MPTP $+m i R-132-3 p$ antagomir diminished the expressions of TNF- $\alpha$ (1.26 \pm 0.21 -fold vs $1.76 \pm 0.30$-fold), IL-1 $\beta$ (1.22 \pm 0.19 -fold vs $1.72 \pm 0.31$-fold), IL-6 (1.34 \pm 0.23 -fold vs $1.81 \pm 0.28$-fold; Fig. $7 F, p<$ $0.05)$. Then, the assessment of the dopaminergic neuron loss by immunofluorescence of tyrosine hydroxylase illustrated that tyrosine hydroxylase-positive neurons in SNc of mice were significantly decreased in the MPTP group $(p<0.001,155.83 \pm 25.97$ vs $621.37 \pm 91.97$ cells $/ \mathrm{mm}^{2}$ ) and markedly increased in the MPTP $+m i R-132-3 p$ antagomir group $\left(401.72 \pm 58.83\right.$ vs $177.83 \pm 32.00 \mathrm{cells} / \mathrm{mm}^{2}$; Fig. $7 \mathrm{G}$, $p<0.01)$. The rotarod test and open field test were used to observe the motor ability of mice. In the rotarod test, mice in the MPTP group showed poor balance and coordination by suppressing the time to $57.38 \pm 12.37 \%$, while mice in the MPTP + miR-132-3p antagomir group exhibited better balance and coordination (84.72 \pm 15.46 vs $52.43 \pm 9.27 \%$; Fig. $7 H, p<$ 0.01). In the open field test, MPTP injection suppressed the spontaneous locomotor activities (whole area: $53.27 \pm 8.91 \%$, central area: $42.35 \pm 6.28 \%$ ), whereas MPTP + miR-132-3p antagomir increased the spontaneous locomotor activities (whole area: $82.47 \pm 13.67 \%$ vs $48.37 \pm 7.21 \%$; central area: $78.61 \pm 11.59 \%$ vs $46.52 \pm 7.38 \%$; Fig. $7 H, p<0.01)$. The above results indicated that MPTP injection may induce dopaminergic neurodegeneration and neuroinflammation of mice, while depletion of $m i R-132-3 p$ may ameliorate the dopaminergic neuron degeneration and neuroinflammation of PD mouse.

\section{Discussion}

Neuroinflammation is a characteristic of neurodegenerative diseases, including PD, in which microglia confer pathogenic and exacerbating effects (Lull and Block, 2010; Zhao et al., 2020). Furthermore, the notable hallmark of PD is the degeneration of dopaminergic neurons in the SNc (Mead et al., 2017). Herein, BV-2 cells and SH-SY5Y were used in current study to explore the effect of miR-132-3p on inflammation of microglial cells and neuronal injury. We have reported that miR-132-3p is abnormally upregulated, a change positively connected with the inflammatory response of microglial cells. We demonstrated that the activated microglial cells caused by $\operatorname{miR}-132-3 p$ leads to increased cell apoptotic rate and diminished viability of neuroblastoma cells. However, miR-132-3p was also reported to alleviate neuron apoptosis and impairments of learning and memory abilities in Alzheimer's disease (Qu et al., 2021). Alzheimer's disease and PD, both belonging to neuro-degenerative diseases: the former is a neurodegenerative brain pathology formed because of piling up of amyloid proteins, development of plaques, and disappearance of neurons (Tufail et al., 2021), while the latter is caused by the loss of dopaminergic neurons in the substantia nigra (Xu et al., 2021). On parallel, the treatment strategies between our two literatures were also different. In our study, LPS induced BV-2 cells were used as inflammatory cell models and the supernatant of BV-2 cell was co-cultured with SH-SY5Y cells. As for the PD rat models, MPTP treatment was given to rats for consecutively $5 \mathrm{~d}$. This discrepancy may be explained by the difference on the disease background and treatment strategy. Similar to our detection, previous studies identified that miR-132-3p was one of the upregulated cimiRNAs in patients with major depression disorder (van den Berg et al., 2020) and was found to be elevated in the serum of patients with mild cognitive impairment (Xie et al., 2015). In addition, we also revealed that GLRX suppresses activation of microglial cells and ameliorates neuronal injury caused by miR-132-3p. Finally, we found that MPTP-induced neuroinflammation and degeneration of dopaminergic neurons in PD mouse models are dramatically attenuated after miR-132-3p downregulation. Thus, our study not only uncovered novel roles for miR132-3p and GLRX in the pathologic abnormalities related to $P D$ but also identified their potential application in the treatment for PD.

Microglial cells are macrophages residing in the brain, which originate from early erythro-myeloid precursors in the embryonic yolk sac (Kanthasamy et al., 2019). Activated microglial cells at the inflammation site promote the release of inflammatory cytokines, thereby intensifying the inflammatory response through activation and recruitment of other cells to the brain lesion (Kim and Joh, 2006). Accumulating evidence proposed that in the process of PD, microglial cells are activated, and then trigger the secretion of a variety of proinflammatory factors, including IL-6, IL-1 $\beta$, and TNF- $\alpha$ (Guan et al., 2017). In an attempt to elucidate the mechanism by which miR-132-3p accelerates the progression of PD, we first investigated whether miR-132-3p affects the activation of microglial cells. Initially, remarkable high expression pattern of miR-132-3p was noticed in midbrain tissues from patients with PD rather than tissues of healthy controls. To this end, LPS was applied to simulate the inflammatory response in BV-2 cells. Herein, results of gain-and lossof-function experiments confirmed that miR-132-3p might likewise contribute to the activation of microglial cells. In our study, knock-down of miR-132-3p can suppress the release of inflammatory cytokines, including TNF- $\alpha$, IL- $1 \beta$, and IL- 6 , while overexpression of miR$132-3 p$ can promote the inflammatory response in BV-2 cells, those results indicated that miR-132 as a driver of microglia proinflammatory responses. Of note, there has been relevant evidence supporting our findings that miR132 confers a pivotal role in intracerebral hemorrhage by regulating inflammation, which is evident from the activation state of microglial cells and the expression of proinflammatory cytokines (Zhang et al., 2017). Furthermore, by using CCK-8 assay and flow cytometry, we discovered that LPS can suppress survival rate of microglial cells and increase cell apoptosis, while further treatment by miR132-3p knock-down partially reverse the LPS induced cell apoptosis and elevate cell survival rate to certain extent. Further measurement showed that activated microglial cells by miR-132-3p may lead to neuronal injury, as evidenced by reinforced cell apoptotic ability and reduced the proliferative ability of SH-SY5Y cells after SH-SY5Y cells 
were cultured with the conditioned medium of BV-2 cells which were transfected with miR-132-3p mimic, which highlighted the role of miR-132-3p in activation of microglial cells and neuronal injury. Interestingly, former work described that the dysregulation of miR-132 leads to the occurrence and exacerbation of PD (Qian et al., 2017). The BACE1-AS/miR-132-3p axis is responsible for the berberine-mitigated neuronal injury in Alzheimer's disease (Ge et al., 2020). Therefore, miR-132-3p may exert a negative effect on PD by inducing neuroinflammation and neuronal injury.

Subsequently, we are prompted to further look into the molecular actions of miR-132-3p in regulating PD by investigating the downstream target. Based on the comprehensive analysis from StarBase, dual-luciferase reporter assay and RIP assay, we identified GLRX as a direct target of miR-132-3p. GLRX is an indispensable thioltransferase whose main function is to remove protein glutathionylation (Burns et al., 2020). Herein, the lowly expressed GLRX was observed in the midbrain tissues of PD patients. Furthermore, analyses of qRTPCR, Western blotting, ELISA, CCK-8 assay and flow cytometry elaborated that miR-132-3p interfered with microglial activation and neuronal injury by targeting GLRX. Our data are in agreement with the earlier findings showing that enhancement of GLRX activity in these brain cells would impede the progression of $P D$ (Gorelenkova Miller and Mieyal, 2019). MPTP is a neurotoxin that results in a profound reduction of striatal dopamine levels and specific loss of dopaminergic neurons in animals (Lee et al., 2019). To further shed light into the relationship between miR-132-3p/GLRX and PD, we used a mouse model of PD stimulated by MPTP. Consistently, mice received MPTP injection had increased inflammatory cytokine release and decreased $\mathrm{TH}$ positive neurons, indicating the neuron loss in MPTP-treated mice. On parallel, elevated miR-132-3p expression and decreased expression of GLRX were also observed in mice in MPTP group, suggesting the possible implication of miR-132-3p in neuron loss of PD mouse. Here, we showed that miR-132-3p downregulation in PD mouse induces alterations in GLRX expression, and miR-132-3p was responsible for the inflammatory response of brain tissues of PD mouse models by modulating GLRX. Additionally, immunofluorescence of Iba1 on detection of microglial activation and immunofluorescence of tyrosine hydroxylase on assessment of dopaminergic neuron loss revealed that depletion of miR-132-3p may alleviate MPTP-induced dopaminergic neurodegeneration and neuroinflammation of PD mouse models. Simultaneously, these findings were further supported by the rotarod test and open field test.

In conclusion, our data suggest that the deficiency of miR-132-3p contributes to ameliorated PD. MiR-132-3p enhances the activation of microglia cells and promotes the release of inflammatory cytokines by targeting GLRX to exert toxic effect on neurons. These findings suggest that targeting neuroprotective pathways controlled by miR-132-3p may represent a potential therapeutic intervention strategy for PD therapy. Further work is required to ascertain whether the protection from PD observed here by silencing of the miR-132-3p is exerted by GLRX, inhibition of microglial activation and dopaminergic neuron loss or perhaps via modulation of other pathways.

\section{References}

Alizadeh R, Kamrava SK, Bagher Z, Farhadi M, Falah M, Moradi F, Boroujeni ME, Soleimani M, Kamyab A, Komeili A (2019) Human olfactory stem cells: as a promising source of dopaminergic neuron-like cells for treatment of Parkinson's disease. Neurosci Lett 696:52-59.

Angelopoulou E, Paudel YN, Piperi C (2019) miR-124 and Parkinson's disease: a biomarker with therapeutic potential. Pharmacol Res 150:104515.

Bassani TB, Vital MA, Rauh LK (2015) Neuroinflammation in the pathophysiology of Parkinson's disease and therapeutic evidence of anti-inflammatory drugs. Arq Neuropsiquiatr 73:616-623.

Burns M, Rizvi SHM, Tsukahara Y, Pimentel DR, Luptak I, Hamburg NM, Matsui R, Bachschmid MM (2020) Role of glutaredoxin-1 and glutathionylation in cardiovascular diseases. Int J Mol Sci 21:6803.

Ge Y, Song X, Liu J, Liu C, Xu C (2020) The combined therapy of berberine treatment with IncRNA BACE1-AS depletion attenuates $A \beta_{25-35}$ induced neuronal injury through regulating the expression of miR-132-3p in neuronal cells. Neurochem Res 45:741-751.

Gorelenkova Miller O, Mieyal JJ (2019) Critical roles of glutaredoxin in brain cells-implications for Parkinson's disease. Antioxid Redox Signal 30:1352-1368.

Guan J, Yang B, Fan Y, Zhang J (2017) GPER agonist G1 attenuates neuroinflammation and dopaminergic neurodegeneration in Parkinson disease. Neuroimmunomodulation 24:60-66.

Hirsch EC, Hunot S (2009) Neuroinflammation in Parkinson's disease: a target for neuroprotection? Lancet Neurol 8:382-397.

Hu YB, Zhang YF, Wang H, J Ren R, Cui HL, Huang WY, Cheng Q, Chen HZ, Wang G (2019) miR-425 deficiency promotes necroptosis and dopaminergic neurodegeneration in Parkinson's disease. Cell Death Dis 10:589.

Ji YF, Wang D, Liu YR, Ma XR, Lu H, Zhang BA (2018) MicroRNA132 attenuates LPS-induced inflammatory injury by targeting TRAF6 in neuronal cell line HT-22. J Cell Biochem 119:5528-5537.

Johnson WM, Yao C, Siedlak SL, Wang W, Zhu X, Caldwell GA, Wilson-Delfosse AL, Mieyal JJ, Chen SG (2015) Glutaredoxin deficiency exacerbates neurodegeneration in C. elegans models of Parkinson's disease. Hum Mol Genet 24:1322-1335.

Kanthasamy A, Jin H, Charli A, Vellareddy A, Kanthasamy A (2019) Environmental neurotoxicant-induced dopaminergic neurodegeneration: a potential link to impaired neuroinflammatory mechanisms. Pharmacol Ther 197:61-82.

Kim YS, Joh TH (2006) Microglia, major player in the brain inflammation: their roles in the pathogenesis of Parkinson's disease. Exp Mol Med 38:333-347.

Krashia P, Cordella A, Nobili A, La Barbera L, Federici M, Leuti A, Campanelli F, Natale G, Marino G, Calabrese V, Vedele F, Ghiglieri V, Picconi B, Di Lazzaro G, Schirinzi T, Sancesario G, Casadei N, Riess O, Bernardini S, Pisani A, et al. (2019) Blunting neuroinflammation with resolvin D1 prevents early pathology in a rat model of Parkinson's disease. Nat Commun 10:3945.

Lee E, Hwang I, Park S, Hong S, Hwang B, Cho Y, Son J, Yu JW (2019) MPTP-driven NLRP3 inflammasome activation in microglia plays a central role in dopaminergic neurodegeneration. Cell Death Differ 26:213-228.

Leinders M, Ûçeyler N, Pritchard RA, Sommer C, Sorkin LS (2016) Increased miR-132-3p expression is associated with chronic neuropathic pain. Exp Neurol 283:276-286.

Li N, Pan X, Zhang J, Ma A, Yang S, Ma J, Xie A (2017) Plasma levels of miR-137 and miR-124 are associated with Parkinson's disease 
but not with Parkinson's disease with depression. Neurol Sci 38:761-767.

Lull ME, Block ML (2010) Microglial activation and chronic neurodegeneration. Neurotherapeutics 7:354-365.

Mead BP, Kim N, Miller GW, Hodges D, Mastorakos P, Klibanov AL, Mandell JW, Hirsh J, Suk JS, Hanes J, Price RJ (2017) Novel focused ultrasound gene therapy approach noninvasively restores dopaminergic neuron function in a rat Parkinson's disease model. Nano Lett 17:3533-3542.

Milanese C, Gabriels S, Barnhoorn S, Cerri S, Ulusoy A, Gornati SV, Wallace DF, Blandini F, Di Monte DA, Subramaniam VN, Mastroberardino PG (2021) Gender biased neuroprotective effect of Transferrin Receptor 2 deletion in multiple models of Parkinson's disease. Cell Death Differ 28:1720-1732.

Postuma RB, Poewe W, Litvan I, Lewis S, Lang AE, Halliday G, Goetz CG, Chan P, Slow E, Seppi K, Schaffer E, Rios-Romenets S, Mi T, Maetzler C, Li Y, Heim B, Bledsoe IO, Berg D (2018) Validation of the MDS clinical diagnostic criteria for Parkinson's disease. Mov Disord 33:1601-1608.

Qazi TJ, Lu J, Duru L, Zhao J, Qing H (2021) Upregulation of mir-132 induces dopaminergic neuronal death via activating SIRT1/P53 pathway. Neurosci Lett 740:135465.

Qian Y, Song J, Ouyang Y, Han Q, Chen W, Zhao X, Xie Y, Chen Y, Yuan W, Fan C (2017) Advances in roles of miR-132 in the nervous system. Front Pharmacol 8:770.

Qu J, Xiong X, Hujie G, Ren J, Yan L, Ma L (2021) MicroRNA-132-3p alleviates neuron apoptosis and impairments of learning and memory abilities in Alzheimer's disease by downregulation of HNRNPU stabilized BACE1. Cell Cycle 20:2309-2312.

Strubberg AM, Madison BB (2017) MicroRNAs in the etiology of colorectal cancer: pathways and clinical implications. Dis Model Mech 10:197-214.

Sveinbjornsdottir S (2016) The clinical symptoms of Parkinson's disease. J Neurochem 139 Suppl 1:318-324.

Tufail AB, Ma YK, Zhang QN, Khan A, Zhao L, Yang Q, Adeel M, Khan R, Ullah I (2021) 3D convolutional neural networks-based multiclass classification of Alzheimer's and Parkinson's diseases using PET and SPECT neuroimaging modalities. Brain Inform 8:23. van den Berg MMJ, Krauskopf J, Ramaekers JG, Kleinjans JCS, Prickaerts J, Briedé JJ (2020) Circulating microRNAs as potential biomarkers for psychiatric and neurodegenerative disorders. Prog Neurobiol 185:101732.

Verma A, Ray A, Bapat D, Diwakar L, Kommaddi RP, Schneider BL, Hirsch EC, Ravindranath V (2020) Glutaredoxin 1 downregulation in the substantia nigra leads to dopaminergic degeneration in mice. Mov Disord 35:1843-1853.

Wang L, Liu J, Liu J, Chen X, Chang M, Li J, Zhou J, Bai C, Song Y (2019) GLRX inhibition enhances the effects of geftinib in EGFRTKI-resistant NSCLC cells through FoxM1 signaling pathway. J Cancer Res Clin Oncol 145:861-872.

Xie B, Zhou H, Zhang R, Song M, Yu L, Wang L, Liu Z, Zhang Q, Cui D, Wang X, Xu S (2015) Serum miR-206 and miR-132 as potential circulating biomarkers for mild cognitive impairment. J Alzheimers Dis 45:721-731.

Xu X, Zhang Y, Kang Y, Liu S, Wang Y, Wang Y, Wang L (2021) LncRNA MIAT inhibits MPP(+)-induced neuronal damage through regulating the miR-132/SIRT1 axis in PC12 cells. Neurochem Res 46:3365-3374.

Yang Z, Li T, Li S, Wei M, Qi H, Shen B, Chang RC, Le W, Piao F (2019) Altered expression levels of microRNA-132 and Nurr1 in peripheral blood of Parkinson's disease: potential disease biomarkers. ACS Chem Neurosci 10:2243-2249.

Ye Y, He X, Lu F, Mao H, Zhu Z, Yao L, Luo W, Sun X, Wang B, Qian C, Zhang Y, Lu G, Zhang S (2018) A lincRNA-p21/miR-181 family feedback loop regulates microglial activation during systemic LPS- and MPTP-induced neuroinflammation. Cell Death Dis 9:803.

Zhang Y, Han B, He Y, Li D, Ma X, Liu Q, Hao J (2017) MicroRNA-132 attenuates neurobehavioral and neuropathological changes associated with intracerebral hemorrhage in mice. Neurochem Int 107:182-190.

Zhao J, Du A, Lu C (2020) Brain gamma rhythm and potential treatment of neurodegenerative disease. J Neurorestoratol 8:26-31. 